TRANSACTIONS OF THE

AMERICAN MATHEMATICAL SOCIETY

Volume 359, Number 8, August 2007, Pages 3609-3645

S 0002-9947(07)04256-0

Article electronically published on March 7, 2007

\title{
ON TORSION-FREE GROUPS WITH FINITE REGULAR FILE BASES
}

\author{
ALEXEY MURANOV
}

\begin{abstract}
The following question was asked by V. V. Bludov in The Kourovka Notebook in 1995: If a torsion-free group $G$ has a finite system of generators $a_{1}, \ldots, a_{n}$ such that every element of $G$ has a unique presentation in the form $a_{1}^{k_{1}} \cdots a_{n}^{k_{n}}$ where $k_{i} \in \mathbb{Z}$, is it true that $G$ is virtually polycyclic? The answer is "not always." A counterexample is constructed in this paper as a group presented by generators and defining relations.
\end{abstract}

\section{INTRODUCTION}

Definition. A group is polycyclic if it has a finite subnormal series with cyclic factors. A group is virtually polycyclic if it has a polycyclic subgroup of finite index.

Subgroups and homomorphic images of polycyclic and virtually polycyclic groups are polycyclic or, respectively, virtually polycyclic. Finitely generated abelian groups are polycyclic. Free groups of rank at least 2 are not virtually polycyclic.

Vasiliy Bludov posed the following question in 1995 (Problem 13.11 in kn; see also Question 6 in [B] $)$ :

If a torsion-free group $G$ has a finite system of generators $a_{1}, \ldots$, $a_{n}$ such that every element of $G$ has a unique presentation in the form $a_{1}^{k_{1}} \cdots a_{n}^{k_{n}}$ where $k_{i} \in \mathbb{Z}$, is it true then that $G$ is virtually polycyclic?

The following theorem answers Bludov's question negatively:

Theorem. Provided $n \geq 63$, there exists a group $G$ and pairwise distinct elements $a_{1}, \ldots, a_{n}$ in $G$ such that:

(0) $G$ is generated by $\left\{a_{1}, \ldots, a_{n}\right\}$;

(1) every $n-21$ elements out of $\left\{a_{1}, \ldots, a_{n}\right\}$ freely generate a free subgroup such that every two elements of this subgroup $F$ are conjugate in $G$ only if they are conjugate in $F$ itself (in particular, $G$ is not virtually polycyclic);

(2) for every element $g$ of $G$, there is a unique $n$-tuple $\left(k_{1}, \ldots, k_{n}\right) \in \mathbb{Z}^{n}$ such that $g=a_{1}^{k_{1}} \cdots a_{n}^{k_{n}}$

(3) $G$ is torsion-free;

Received by the editors March 4, 2005

2000 Mathematics Subject Classification. Primary 20F05; Secondary 20 F06.

Key words and phrases. Group presentation, van Kampen's lemma, diagram with selection, S-diagram, virtually polycyclic group, boundedly generated group, file basis.

This work was supported in part by the NSF grant DMS 0245600 of Alexander Ol'shanskiy and Mark Sapir.

(c)2007 American Mathematical Society Reverts to public domain 28 years from publication 
(4) $G$ is the direct limit of a sequence of hyperbolic groups with respect to a family of surjective homomorphisms;

(5) $G$ is recursively presented and has solvable word and conjugacy problems.

(The direct limit in condition (4) is the direct limit in the sense of Bourbaki; see "Algebra I.")

This theorem is proved in the end of Section 5 of this paper.

In terms of file bases (see definitions in [B1]), item (2) of the theorem states that the elements $a_{1}, \ldots, a_{n}$ in this order form a regular file basis of $G$. The argument below shows that every group with a 2-element file basis is polycyclic (therefore, the assumption " $n \geq 63$ " cannot be replaced with " $n \geq 2$ ").

Every group that has an $n$-element file basis is $n$-boundedly generated (i.e., is the product of an $n$-tuple of its cyclic subgroups). The following two theorems (see [I] and [LeR] together yield that every 2-boundedly generated group is polycyclic:

Theorem (Itô, 1955). If a group $G$ has abelian subgroups $A$ and $B$ such that $G=A B$, then $G$ is metabelian.

Theorem (Lennox, Roseblade, 1980). If a soluble group $G$ has polycyclic subgroups $A$ and $B$ such that $G=A B$, then $G$ is polycyclic.

Some examples of non-polycyclic groups with finite regular file bases and with torsion are known. It is shown in Bl] (see Example 3 therein) that the semidirect product of the rank- $n$ free abelian group $\mathbb{Z}^{n}$ with the symmetric group $S_{n}$ defined via the action of $S_{n}$ on $\mathbb{Z}^{n}$ by naturally permuting the standard generators has an $n$-element regular file basis of infinite-order elements. If $n \geq 5$, then such a semidirect product is virtually polycyclic but not polycyclic.

The primary tool used to establish properties of the group (or rather the series of groups) constructed in this paper is the Main Theorem in [Mu.

\section{Group construction}

Take an arbitrary integer $n \geq 63$. Choose a positive $\lambda_{1}<1$ such that

$$
\left(4+\frac{2 n \lambda_{1}}{1-\lambda_{1}}\right) \lambda_{1} \leq \frac{1}{n} .
$$

(It suffices if, for example, $0<\lambda_{1} \leq 1 /(5 n)$.)

Let $\mathfrak{A}=\left\{x_{1}, \ldots, x_{n}\right\}$ be an $n$-letter alphabet. Call a group word $w$ over $\mathfrak{A}$ regular if it has the form $x_{1}^{k_{1}} x_{2}^{k_{2}} \cdots x_{n}^{k_{n}}$ where $k_{i} \in \mathbb{Z}$. (In particular, regular group words are cyclically reduced.) Call a group word $w$ counter-regular if $w^{-1}$ is regular. The empty word is both regular and counter-regular. So is every group word that is a letter power.

Impose an order on the set of all reduced group words in the alphabet $\mathfrak{A}$ to make it order-isomorphic with the set of natural numbers. For example, use the deg-lex order: $x_{1}, x_{1}^{-1}, x_{2}, \ldots, x_{n}^{-1}, x_{1}^{2}, x_{1} x_{2}, x_{1} x_{2}^{-1}, \ldots$ Define a sequence of sets $\left\{\mathcal{R}_{i}\right\}_{i=0}^{+\infty}$ inductively.

First, let $\mathcal{R}_{0}=\emptyset$.

Second, if $i>0$ and every group word over $\mathfrak{A}$ equals a regular word modulo the relations $r=1, r \in \mathcal{R}_{i-1}$, then let $\mathcal{R}_{i}=\mathcal{R}_{i-1}$.

Last, if $i>0$ and some group word over $\mathfrak{A}$ is not equal to any regular word modulo the relations $r=1, r \in \mathcal{R}_{i-1}$, then let $w_{i}$ be the least reduced group word (with respect to the chosen order) that does not start with $x_{1}^{ \pm 1}$, does not end with 
$x_{n}^{ \pm 1}$, and is not equal to any regular word modulo the relations $r=1, r \in \mathcal{R}_{i-1}$ (clearly such $w_{i}$ exists). Choose a natural number $m_{i}$ such that

$$
\begin{gathered}
i>j \Rightarrow m_{i} \neq m_{j}, \\
i>j \Rightarrow n m_{i}+\left|w_{i}\right| \geq n m_{j}+\left|w_{j}\right|, \\
\lambda_{1}\left(n m_{i}+\left|w_{i}\right|\right) \geq\left|w_{i}\right| .
\end{gathered}
$$

(These conditions may be satisfied by choosing a sufficiently large $m_{i}$.) Define

$$
r_{i}=x_{1}^{m_{i}} x_{2}^{m_{i}} \cdots x_{n}^{m_{i}} w_{i}^{-1}
$$

and let $\mathcal{R}_{i}=\mathcal{R}_{i-1} \cup\left\{r_{i}\right\}$. Note that $\left|r_{i}\right|=n m_{i}+\left|w_{i}\right|$.

Eventually, let

$$
\mathcal{R}=\bigcup_{i=1}^{+\infty} \mathcal{R}_{i}
$$

and let $G$ be the group defined by the presentation $\langle\mathfrak{A} \| r=1, r \in \mathcal{R}\rangle$.

Observe that all elements of $\mathcal{R}$ are cyclically reduced.

Along with $\left\{\mathcal{R}_{i}\right\}_{i=0}^{+\infty}$, the sequences $\left\{w_{i}\right\}_{i=1, \ldots},\left\{m_{i}\right\}_{i=1, \ldots}$, and $\left\{r_{i}\right\}_{i=1, \ldots}$, infinite or finite, have been constructed.

Note that the above construction scheme does not specify $\mathcal{R}$ uniquely and is flexible as to the choice of $\left\{w_{i}\right\}_{i=1, \ldots}$ and $\left\{m_{i}\right\}_{i=1, \ldots}$.

\section{Definitions}

Most definitions needed for reading this paper may be found in $\mathrm{Mu}$. Standard terms are also defined in $\mathrm{LyS}, \mathrm{ChCoH}, \mathrm{O} 1$ in similar ways. For instance, geometric definitions of maps and diagrams are given in O1, and combinatorial ones in $\mathrm{Mu}$. Some definitions are reviewed in this section.

If $X$ is a set, then $\|X\|$ denotes the cardinality of $X$.

A graph $\Gamma$ is an ordered pair $(\Gamma(0), \Gamma(1))$ where $\Gamma(0)$ is the set of vertices of $\Gamma$, and $\Gamma(1)$ is its set of edges. It will always be assumed that $\Gamma(0)$ is nonempty. In the context of this paper, graph is a synonym of combinatorial 1-complex. A combinatorial 2-complex $\Phi$ is an ordered triple $(\Phi(0), \Phi(1), \Phi(2))$ where $\Phi(0), \Phi(1)$, and $\Phi(2)$ are its sets of vertices, edges, and faces, respectively.

If $v$ is a vertex of a complex $\Gamma$, then the number of all edges of $\Gamma$ incident to $v$ plus the number of all loops incident to $v$ (thus loops are counted twice) is called the degree of the vertex $v$ and is denoted by $d(v)$ or $d_{\Gamma}(v)$.

Here follow several definitions related to paths in combinatorial complexes.

The length of a path $p$ is denoted by $|p|$. A cyclic path is a path whose terminal and initial vertices coincide. A cycle is the set of all cyclic shifts of a cyclic path. The cycle represented by a cyclic path $p$ shall be denoted by $\langle p\rangle$. The length of a cycle $c$, denoted by $|c|$, is the length of an arbitrary representative of $c$. A trivial cycle is a cycle of length zero. A path $p$ is a subpath of a cycle $c$ if for some representative $r$ of $c$ and for some natural $n, p$ is a subpath of $r^{n}$ (i.e., of the product of $n$ copies of $r$ ).

A path is reduced if it does not have a subpath of the form $e e^{-1}$ where $e$ is an oriented edge. A cyclic path is cyclically reduced if it is reduced, and its first oriented edge is not inverse to its last oriented edge. (For example, all trivial paths are cyclically reduced.) A cycle is reduced if it consists of cyclically reduced cyclic paths. A path is simple if it is nontrivial, reduced, and none of its intermediate 
vertices appears in it more than once. A cycle is simple if it consists of simple cyclic paths. A path is maximal in some set of paths if it is not a proper subpath of any other path in this set.

An oriented arc in a complex $\Gamma$ is a simple path whose all intermediate vertices have degree 2 in $\Gamma$. A non-oriented arc, or simply an arc, is defined as a pair of mutually inverse oriented arcs. Edges and oriented edges shall be viewed as special cases of arcs and oriented arcs, respectively. Two arcs, or oriented arcs, are said to overlap if they have common edges. A set of arcs, or oriented arcs, is called non-overlapping if no two distinct elements of this set overlap. An arc $u$ lies on a path $p$ if at least one of the oriented arcs associated with $u$ is a subpath of $p$.

The remaining definitions concern maps and diagrams.

Every oriented face of a 2-complex has a unique boundary cycle. Every (nonoriented) face of an oriented combinatorial surface has a unique contour, which is defined as the boundary cycle of the corresponding oriented face. The contour of a face $\Pi$ shall be denoted by $\partial \Pi$. Thus the formula $\langle p\rangle=\partial \Pi$ means that the path $p$ is a representative of the contour of the face $\Pi$. The degree of a face $\Pi$ is the length of the contour of $\Pi$, denoted by $|\partial \Pi|$.

A map is a connected subcomplex of an oriented combinatorial sphere together with a set of cycles called its contours (see $[\mathrm{Mu}$ for a precise definition). In every map, each oriented edge appears either in the contour of some face of the map or in a contour of the map. The union of all contours of a map $\Delta$ shall be denoted by $\partial \Delta$. A map without faces is called degenerate. A map is simple if all of its contours are simple cycles without common vertices. A disc map is a map with exactly one contour. A disc map is simple if and only if its underlying 2-complex is a combinatorial disc. An annular map is a map with exactly two contours.

The contours of faces in a disc map shall be thought of as oriented counterclockwise, and the contour of the map itself shall be thought of as oriented clockwise (this is consistent with the way disc maps are pictured in this paper).

A selection on a face $\Pi$ of a map $\Delta$ is a set of nontrivial reduced subpaths of the contour of $\Pi$ closed under taking nontrivial subpaths. A selection on a map $\Delta$ is a set of nontrivial reduced subpaths of the contours of faces of $\Delta$ closed under taking nontrivial subpaths. An $S$-map is a map with selection. A path in an S-map is selected if it belongs to the selection. A path $p$ is double-selected if both $p$ and $p^{-1}$ are selected. An arc is double-selected if both corresponding oriented arcs are selected. An external arc is selected if one of the corresponding oriented arcs is selected.

A diagram over a presentation $\langle\mathfrak{B} \| \mathcal{S}\rangle$ is a map together with a function which labels every oriented edge of this map with a letter from $\mathfrak{B}^{ \pm 1}$ so that mutually inverse oriented edges are labelled with mutually inverse group letters, and the contour of every face has a representative "labelled" with an element of $\mathcal{S}^{ \pm 1}$. A diagram together with a selection on the underlying map is called an S-diagram.

In a diagram over $\langle\mathfrak{B} \| \mathcal{S}\rangle$, let the label of an oriented edge be the element of $\mathfrak{B}^{ \pm 1}$ that labels this oriented edge, and let the label of a path be the group word that "reads" on this path. Let the label of a (non-oriented) edge be the letter $x$ of $\mathfrak{B}$ such that $x^{+1}$ and $x^{-1}$ label the two corresponding oriented edges. If $q$ is an oriented edge or a path, the label $q$ shall be denoted by $\ell(q)$.

Sometimes it is convenient to distinguish between a letter $x$ of the alphabet $\mathfrak{B}$ and the corresponding letter of the group alphabet $\mathfrak{B}^{ \pm 1}$ denoted by $x^{+1}$ or simply 
by $x$. Call letters of the alphabet $\mathfrak{B}$ basic letters and letters of the group alphabet $\mathfrak{B}^{ \pm 1}$ group letters. Thus, the basic letter $x_{1}$, the group letter $x_{1}$, and the one-letter group word $x_{1}$ are three different things. The labels of edges of a diagram are basic letters, but the labels of oriented edges are group letters. Say that a group word $w$ has a basic letter $x$ in it if at least one of the two group letters $x^{ \pm 1}$ occurs in $w$.

The mirror copy of a map or diagram is the map or diagram, respectively, with the same underlying 2-complex, and the same labelling function in the case of diagram, but with the opposite orientation of all the faces and contours.

According to van Kampen's lemma (see [01]), a relation $w=1$ is a consequence of a set of relations $\{r=1 \mid r \in \mathcal{S}\}$ if and only if there exists a disc diagram $\Delta$ over $\langle\mathfrak{B} \| \mathcal{S}\rangle$, where $\mathfrak{B}$ is an appropriate alphabet, such that the label of some representative of $\partial \Delta$ is $w$. Such a diagram is called a deduction diagram for the relation $w=1$.

Call a pair of distinct faces $\left\{\Pi_{1}, \Pi_{2}\right\}$ in a diagram $\Delta$ immediately cancellable if there exist paths $p_{1}$ and $p_{2}$ with a nontrivial common initial subpath such that $\left\langle p_{1}\right\rangle=\partial \Pi_{1},\left\langle p_{2}^{-1}\right\rangle=\partial \Pi_{2}$, and $\ell\left(p_{1}\right)=\ell\left(p_{2}\right)$. A diagram without immediately cancellable pairs of faces is called weakly reduced. (In $[\mathrm{Mu}$ immediately cancellable pairs of faces and weakly reduced diagrams are called simply cancellable and reduced, respectively.) If there exists a deduction diagram for a relation $w=1$ over a presentation $\langle\mathfrak{B} \| \mathcal{S}\rangle$, then such a diagram may be chosen weakly reduced, and even reduced in the sense of [01, which is a stronger property (take an arbitrary deduction diagram and "eliminate" immediately cancellable pairs).

Call a group presentation $\langle\mathfrak{B} \| \mathcal{S}\rangle$ strongly aspherical if no spherical diagram over $\langle\mathfrak{B} \| \mathcal{S}\rangle$ is weakly reduced.

\section{The Main Lemma}

This paper relies on a technical result of $\mathrm{Mu}$ called there "the Main Theorem." In fact, it suffices to use a simplified form of that result, which is stated in this section as the Main Lemma.

"Inductive Lemmas" and "the Main Theorem" in this section refer to the corresponding statements in $\mathrm{Mu}$. Because they are not used outside of this section, because their statements involve properties of maps that are not defined in this paper, and because the Main Lemma may be viewed as a particular case of the Main Theorem, the Main Theorem and Inductive Lemmas are not stated here.

Definition. Call a map $\Delta$ semisimple if every edge of $\Delta$ is incident to a face of $\Delta$.

Definition. Let $\Delta$ be a semisimple S-map. Let $S$ be the number of selected external edges of $\Delta$. Let $\mu$ be a real number. Then the S-map $\Delta$ is said to satisfy the condition $\mathcal{X}(\mu)$ if

$$
S \geq\|\Delta(1)\|-\mu \sum_{\Pi \in \Delta(2)}|\partial \Pi|
$$

Note that the definition of the condition $\mathcal{X}$ in $[\mathrm{Mu}$, given there only for simple $\mathrm{S}$-maps, is extended here to all semisimple S-maps.

Proposition 4.1. Let $\Delta$ be an $S$-map, and let $\mu$ be a real number. Suppose every maximal semisimple $S$-submap of $\Delta$ satisfies the condition $\mathcal{X}(\mu)$. Let $S$ be the 
number of selected external edges of $\Delta$. Then

$$
S \geq(1-2 \mu) \sum_{\Pi \in \Delta(2)}|\partial \Pi| .
$$

Proof. Consider an arbitrary maximal semisimple S-submap $\Delta^{\prime}$ of $\Delta$. Let $S^{\prime}$ be the number of selected external edges of $\Delta^{\prime}$. Let $\Sigma^{\prime}$ be the sum of the degrees of all the faces of $\Delta^{\prime}$. Then

$$
S^{\prime}=2 S^{\prime}-S^{\prime} \geq 2\left\|\Delta^{\prime}(1)\right\|-2 \mu \Sigma^{\prime}-S^{\prime} \geq S^{\prime}+\Sigma^{\prime}-2 \mu \Sigma^{\prime}-S^{\prime}=(1-2 \mu) \Sigma^{\prime} .
$$

Now, consider $\Delta$ itself. Remove from the underlying 2-complex of $\Delta$ all edges that are not incident to any face. All connected components of the remaining 2complex (some, possibly, consisting of just one vertex) define semisimple S-submaps of $\Delta$. These S-submaps are precisely all maximal semisimple S-submaps of $\Delta$. Therefore, every selected external edge of $\Delta$, as well as every face of $\Delta$, belongs to exactly one maximal semisimple S-submap of $\Delta$. Hence, the desired inequality for $\Delta$ may be obtained by adding up the analogous inequalities for all of its maximal semisimple S-submaps.

Although it takes little effort to derive the Main Lemma formulated below from the Main Theorem, it will be even easier if a modified version of the Main Theorem with slightly weakened hypotheses is used instead. It can be observed that the Main Theorem will still hold true if "simple S-map" is replaced with "semisimple S-map" in the hypotheses. Indeed, analysis of the proofs of Inductive Lemmas shows that their statements can be modified in the following way without turning them false:

(1) The hypotheses of Inductive Lemma 1 may be weakened by requiring the condition $\mathcal{X}(\mu)$ only from all simple disc S-submaps of $\Delta$ all of whose edges are internal in $\Delta$, rather than from all proper simple disc S-submaps.

(2) The hypotheses of Inductive Lemma 2 may be weakened by requiring the S-map $\Delta$ to be only semisimple, rather than simple.

The original proofs with a few obvious changes apply to the modified lemmas.

The statement obtained from the Main Theorem by replacing "simple" with "semisimple" shall be referred to as the Modified Main Theorem. The original proof of the Main Theorem applies to the Modified Main Theorem if Inductive Lemmas, used in the proof, are modified as described. (The condition $\mathcal{X}$ in the conclusions of the Modified Main Theorem and modified Inductive Lemma 2 shall be understood in the above-defined broader sense.)

Remark 4.1. The Modified Main Theorem can also be obtained as a corollary of the Main Theorem by "cutting" a given semisimple S-map into its "simple components."

Definition. Let $\Delta$ be an S-map. Let $\lambda_{1}, \lambda_{2} \in[0,1]$. The S-map $\Delta$ is said to satisfy the condition $\mathcal{B}\left(\lambda_{1}, \lambda_{2}\right)$ if it satisfies the following three conditions:

$\mathcal{B}_{0}$ : For each face $\Pi$ of $\Delta$, the contour of $\Pi$ has at least one selected subpath and at most one maximal selected subpath (note that if all nontrivial subpaths are selected, then there is no maximal selected subpath).

$\mathcal{B}_{1}\left(\lambda_{1}\right)$ : For each face $\Pi$ of $\Delta$, there is a selected subpath of $\partial \Pi$ of length at least $\left(1-\lambda_{1}\right)|\partial \Pi|$.

$\mathcal{B}_{2}\left(\lambda_{2}\right)$ : For each face $\Pi$ of $\Delta$, the length of every double-selected arc incident to $\Pi$ is at most $\lambda_{2}|\partial \Pi|$. 
A map $\Delta$ is said to satisfy the condition $\mathcal{B}\left(\lambda_{1}, \lambda_{2}\right)$ if there exists a selection on $\Delta$ such that $\Delta$ with this selection satisfies $\mathcal{B}\left(\lambda_{1}, \lambda_{2}\right)$.

For all admissible values of $\lambda_{1}$ and $\lambda_{2}$, the condition $\mathcal{B}\left(\lambda_{1}, \lambda_{2}\right)$ is equivalent to the condition $\mathcal{A}\left(1,1,1 ; \lambda_{1}, \lambda_{2}, 0,0\right)$ in $\underline{\mathrm{Mu}}$.

Main Lemma. Let $\Delta$ be an $S$-map with at most 3 contours. Let $\lambda_{1}, \lambda_{2} \in[0,1]$. Suppose $2 \lambda_{1}+13 \lambda_{2}<1$ and $\Delta$ satisfies $\mathcal{B}\left(\lambda_{1}, \lambda_{2}\right)$. Let $S$ be the number of selected external edge of $\Delta$, and let $\mu=\lambda_{1}+5 \lambda_{2}$. Then

$$
S \geq(1-2 \mu) \sum_{\Pi \in \Delta(2)}|\partial \Pi| .
$$

Proof. Every maximal semisimple S-submap of $\Delta$ has at most 3 contours (see the description of maximal semisimple S-submaps given in the proof of Proposition 4.1) and satisfies the condition $\mathcal{B}\left(\lambda_{1}, \lambda_{2}\right)$. By the Modified Main Theorem, and Remarks 6.3 and $6.4 \mathrm{in}[\mathrm{Mu}$, all maximal semisimple S-submaps of $\Delta$ satisfy the condition $\mathcal{X}(\mu)$. The desired inequality now follows from Proposition 4.1.

\section{Properties of the Group}

In this section some properties of the above-defined group $G$ are established. In particular, it is shown that $G$ is an example that provides the negative answer to Bludov's question.

Adopt the notation of Section 2. In particular, define $n$ and $\lambda_{1}$ the same way. Let $\lambda_{2}=2 / n, \mu=\lambda_{1}+5 \lambda_{2}$. Then

$$
2 \lambda_{1}+13 \lambda_{2}<1
$$

and

$$
1-2 \mu-2 \lambda_{1}-\frac{2 n \lambda_{1}^{2}}{1-\lambda_{1}} \geq 1-\frac{21}{n} .
$$

In particular $\mu<1 / 2$. It is well known that $21 \times 3=63$; this explains why 63 .

Inequality (2.4) is equivalent to

$$
m_{i} \geq \frac{1-\lambda_{1}}{n}\left(n m_{i}+\left|w_{i}\right|\right) .
$$

Combining (2.4), (5.3) and (2.3), obtain

$$
j \leq i \Rightarrow\left|w_{j}\right| \leq \frac{n \lambda_{1}}{1-\lambda_{1}} m_{i} .
$$

Inequality (2.1) implies that $\lambda_{1}<1 /(4 n)<1 /(2 n+1)$. It follows from this and from (5.4) that

$$
j \leq i \Rightarrow\left|w_{j}\right|<\frac{m_{i}}{2} .
$$

If $w$ is a group word over $\mathfrak{A}$, let $[w]_{G},[w]_{\mathcal{R}}$, or simply $[w]$, denote the element of $G$ represented by $w$. Let $a_{1}, \ldots, a_{n}$ be the elements of $G$ represented by the one-letter group words $x_{1}, \ldots, x_{n}$, respectively (in terms of "brackets," $a_{i}=\left[x_{i}\right]$ ).

In this section a selection on a diagram $\Delta$ is called special if the contour of every face $\Pi$ of $\Delta$ has two subpaths $s$ and $t$ such that:

- $\langle s t\rangle=\partial \Pi$

- $s$ is the only maximal selected subpath of $\partial \Pi$; 
- there is $m \in \mathbb{N}$ such that either $\ell(s)=x_{1}^{m} x_{2}^{m} \cdots x_{n}^{m}$ or $\ell(s)=x_{n}^{-m} x_{n-1}^{-m} \cdots$ $x_{1}^{-m}$

- $|s|>\frac{n}{2 n-2}|\partial \Pi| \quad$ (note that $\left.|\partial \Pi|=|s|+|t|\right)$.

On every diagram $\Delta$ over $\langle\mathfrak{A} \| \mathcal{R}\rangle$, there exists a unique special selection. (In fact, if $\Delta$ is a diagram over an arbitrary group presentation, and a special in the above sense selection on $\Delta$ exists, then it is unique.) A diagram over $\langle\mathfrak{A} \| \mathcal{R}\rangle$ together with a special selection shall be called a special S-diagram. Note that if $s$ is the maximal selected subpath of the contour of a face $\Pi$ of a special S-diagram over $\langle\mathfrak{A} \| \mathcal{R}\rangle$, then $|s| \geq\left(1-\lambda_{1}\right)|\partial \Pi|$, which is stronger than the inequality in the definition of a special selection.

If $\Delta$ is a diagram over $\langle\mathfrak{A} \| \mathcal{R}\rangle$, and $\Pi$ is a face of $\Delta$, then define the rank of the face $\Pi$ to be such $j$ that the label of some representative of $\partial \Pi$ is $r_{j}^{ \pm 1}$. Clearly, the rank of a face is well-defined. Let the rank of a face $\Pi$ be denoted by $\operatorname{rank}(\Pi)$. Note that if $\operatorname{rank}\left(\Pi_{1}\right) \geq \operatorname{rank}\left(\Pi_{2}\right)$, then $\left|\partial \Pi_{1}\right| \geq\left|\partial \Pi_{2}\right|$. The ranks of two faces in a special S-diagram over $\langle\mathfrak{A} \| \mathcal{R}\rangle$ are equal if and only if the lengths of the maximal selected subpaths of their contours are equal (follows from (2.2)).

Proposition 5.1. Every weakly reduced special $S$-diagram over $\langle\mathfrak{A} \| \mathcal{R}\rangle$ satisfies the condition $\mathcal{B}\left(\lambda_{1}, \lambda_{2}\right)$.

Proof. Let $\Delta$ be a weakly reduced special S-diagram over $\langle\mathfrak{A} \| \mathcal{R}\rangle$. Clearly, $\Delta$ satisfies $\mathcal{B}_{0}$.

If $\Pi$ is a face of $\Delta, s$ is the maximal selected subpath of $\partial \Pi$, and $t$ is the path such that $\langle s t\rangle=\partial \Pi$, then $\ell(s)=\left(x_{1}^{m_{j}} x_{2}^{m_{j}} \cdots x_{n}^{m_{j}}\right)^{ \pm 1}$ and $\ell(t)=w_{j}^{\mp 1}$ where $j=\operatorname{rank} \Pi$. Since $\left|w_{j}\right| \leq \lambda_{1}\left|r_{j}\right|$ (see (2.4)), have that $|s| \geq\left(1-\lambda_{1}\right)|\partial \Pi|$. Hence, $\Delta$ satisfies $\mathcal{B}_{1}\left(\lambda_{1}\right)$.

If $u$ is a double-selected oriented arc, then $\ell(u)$ is a subword of a word of the form $x_{l}^{m} x_{l+1}^{m}$ or $x_{l+1}^{-m} x_{l}^{-m}$ (since $\Delta$ is weakly reduced). Therefore, if such a $u$ is a subpath of the contour of a face $\Pi$, then $|u|<(2 / n)|\partial \Pi|=\lambda_{2}|\partial \Pi|$. Hence, $\Delta$ satisfies $\mathcal{B}_{2}\left(\lambda_{2}\right)$.

Corollary 1. If $\Delta$ is a weakly reduced special $S$-diagram over $\langle\mathfrak{A} \| \mathcal{R}\rangle$ with at most 3 contours, and $S$ is the number of selected external edge of $\Delta$, then

$$
S \geq(1-2 \mu) \sum_{\Pi \in \Delta(2)}|\partial \Pi| .
$$

Proof. Follows from the proposition and the Main Lemma.

Corollary 2. The group presentation $\langle\mathfrak{A} \| \mathcal{R}\rangle$ is strongly aspherical in the sense that no spherical diagram over $\langle\mathfrak{A} \| \mathcal{R}\rangle$ is weakly reduced.

Proof. Every weakly reduced special spherical S-diagram over $\langle\mathfrak{A} \| \mathcal{R}\rangle$ has at least $(1-2 \mu) \cdot 2$ selected external edges and therefore does not exist $(\mu<1 / 2)$.

Proposition 5.2. Let $\Delta$ be a special nondegenerate $S$-diagram over $\langle\mathfrak{A} \| \mathcal{R}\rangle$. Let $\mathfrak{B}$ be an arbitrary nonempty subset of $\mathfrak{A}$, and let $k=|\mathfrak{B}|$. Let $S$ be the number of selected external edges of $\Delta$ whose labels are in $\mathfrak{B}$. Then

$$
S<\frac{k}{n} \sum_{\Pi \in \Delta(2)}|\partial \Pi| .
$$


Proof. If $\Pi$ is a face of $\Delta$, and $s$ is the maximal selected subpath of $\partial \Pi$, then $\ell(s)=$ $\left(x_{1}^{m_{j}} x_{2}^{m_{j}} \cdots x_{n}^{m_{j}}\right)^{ \pm 1}$ where $j=\operatorname{rank} \Pi$. Therefore, the number of selected external edges of $\Delta$ incident to $\Pi$ whose labels are in $\mathfrak{B}$ is at most $(k / n)|s|<(k / n)|\partial \Pi|$. Since this is true for every face $\Pi$ of $\Delta$, the desired inequality follows.

Definition. Let $\langle\mathfrak{B} \| \mathcal{S}\rangle$ be a finite group presentation. A function $f: \mathbb{N} \rightarrow \mathbb{R}$ is called an isoperimetric function of $\langle\mathfrak{B} \| \mathcal{S}\rangle$ if for every group word $w$ over $\mathfrak{B}$ equal to 1 modulo the relations $r=1, r \in \mathcal{S}$, there exists a disc diagram $\Delta$ over $\langle\mathfrak{B} \| \mathcal{S}\rangle$ with at most $f(|w|)$ faces such that $w$ is the label of some representative of $\partial \Delta$. The minimal isoperimetric function of $\langle\mathfrak{B} \| \mathcal{S}\rangle$ is called the Dehn function of $\langle\mathfrak{B} \| \mathcal{S}\rangle$.

Proposition 5.3. If $\langle\mathfrak{B} \| \mathcal{S}\rangle$ is a finite subpresentation of $\langle\mathfrak{A} \| \mathcal{R}\rangle(\mathfrak{B}$ is a subset of $\mathfrak{A}$, and $\mathcal{S}$ is a subset of $\mathcal{R}$ ), then the function $f: \mathbb{N} \rightarrow \mathbb{R}$ given by the formula

$$
f(k)=\frac{k}{1-2 \mu}
$$

is an isoperimetric function of $\langle\mathfrak{B} \| \mathcal{S}\rangle$ (recall that $\mu<1 / 2)$.

Proof. If $\Delta$ is a weakly reduced disc diagram over $\langle\mathfrak{B} \| \mathcal{S}\rangle$, and $\langle q\rangle=\partial \Delta$, then, as follows from Corollary 1 of Proposition 5.1,

$$
|q| \geq(1-2 \mu) \sum_{\Pi \in \Delta(2)}|\partial \Pi| \geq(1-2 \mu)\|\Delta(2)\| .
$$

Hence, $\|\Delta(2)\| \leq f(|q|)$.

Corollary. Every finite subpresentation of $\langle\mathfrak{A} \| \mathcal{R}\rangle$ presents a hyperbolic group.

Proof. Use the characterization of hyperbolic groups in terms of isoperimetric functions of their finite presentations. According to Theorem 2.5, Theorem 2.12, and Corollary of the latter in $\mathrm{ABC}+$, a group is hyperbolic if and only if it has a finite presentation with a linear isoperimetric function. (Note that in $\mathrm{ABC}+$ all isoperimetric functions in the sense of the last definition are called "Dehn functions.")

Let deg-lex be the order on the set of all group words over $\mathfrak{A}$ described as follows. Two group words are compared first by length, and second alphabetically according to the following order on the group letters: $x_{1}<x_{1}^{-1}<x_{2}<\cdots<x_{n}<x_{n}^{-1}$. For example, $x_{n} x_{n}<x_{1} x_{1} x_{1}$ and $x_{1} x_{2} x_{3}<x_{1} x_{3} x_{2}$.

Proposition 5.4. If the order on reduced group words used in Section 2 for choosing $\left\{w_{i}\right\}_{i=1, \ldots}$ is deg-lex, $N$ is a positive integer, and $m_{i}=N\left|w_{i}\right|+i$ for every $i$ for which $r_{i}$ is defined, then the presentation $\langle\mathfrak{A} \| \mathcal{R}\rangle$ is recursive.

Proof. If the presentation $\langle\mathfrak{A} \| \mathcal{R}\rangle$ is finite, then it is recursive. (It will be shown in Section 6 that it cannot be finite.) Now, assume that the presentation is infinite.

Let $C$ be the set of all 4 -tuples $(\mathcal{S}, E, u, v)$ such that $\mathcal{S}$ is a finite set of group words over $\mathfrak{A}, E$ is a rational number, $u$ and $v$ are group words over $\mathfrak{A}$, and there exists a disc diagram over $\langle\mathfrak{A} \| \mathcal{S}\rangle$ with at most $E$ edges such that the label of some representative of its contour is $u v^{-1}$. The set $C$ is recursive.

Let $q$ be a rational number such that $q \geq 1 /(1-2 \mu)$. Let $D$ be the set of all 3 -tuples $(\mathcal{S}, u, v)$ such that

$$
\left(\mathcal{S}, \frac{1+q L}{2}(|u|+|v|), u, v\right) \in C
$$


if $L$ is the maximum of the lengths of the elements of $\mathcal{S}$. The set $D$ is recursive. This set describes consequences of a given finite set of relations as explained below.

Let $f$ be the function $\mathbb{N} \rightarrow \mathbb{R}, k \mapsto q k$. Consider an arbitrary finite $\mathcal{S}$ such that $f$ is an isoperimetric function of $\langle\mathfrak{A} \| \mathcal{S}\rangle$. Let $u$ and $v$ be arbitrary group words over $\mathfrak{A}$. Then the relation $u=v$ is a consequence of the relations of $\langle\mathfrak{A} \| \mathcal{S}\rangle$ if and only if $(\mathcal{S}, u, v) \in D$.

Consider the following algorithm:

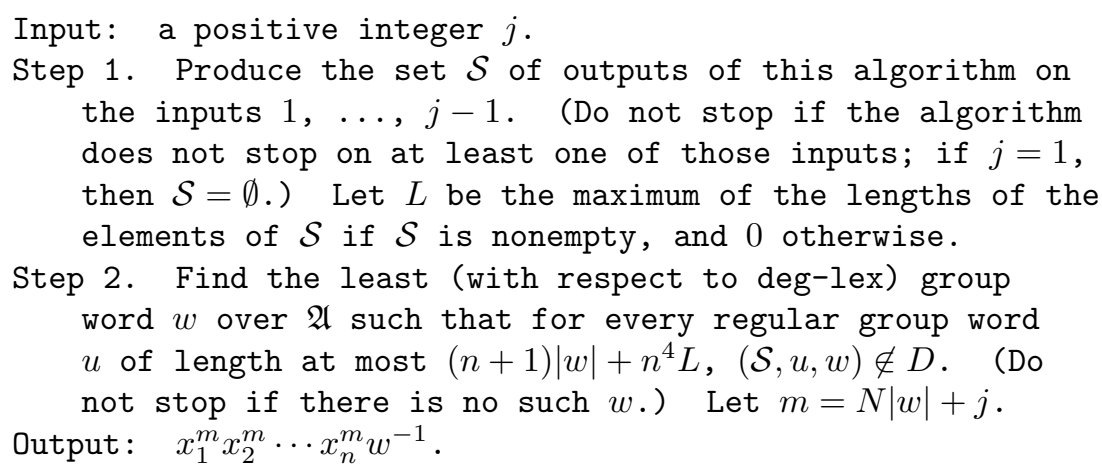

It shall be shown by induction that on every input $j \in \mathbb{N}$, the algorithm stops and gives $r_{j}$ as the output. It will follow that $\mathcal{R}$ is recursive because the length of $r_{j}$ is an increasing function of $j$.

Take an arbitrary $k \in \mathbb{N}$. If $k>1$, assume that it is already proved that for every positive integer $j<k$, the algorithm gives $r_{j}$ as the output on the input $j$. Consider the work of the algorithm on the input $k$.

By the inductive assumption, the set $\mathcal{S}$ produced in Step 1 coincides with $\mathcal{R}_{k-1}$. In particular, $f$ is an isoperimetric function of $\langle\mathfrak{A} \| \mathcal{S}\rangle$. Note that $L=\left|r_{k-1}\right|$ if $k>1$, and $L=0$ if $k=1$.

By the choice of $w_{k}$, this word is not equal to any regular group word modulo the relations of $\langle\mathfrak{A} \| \mathcal{S}\rangle$. Therefore, Step 2 of the algorithm is carried out in finite time, and the obtained group word $w$ is not greater than $w_{k}$ with respect to the deg-lex order.

To complete the inductive step and the proof of this proposition, it suffices to show that the group word $w$ obtained in Step 2 is $w_{k}$. Suppose $w$ is distinct from $w_{k}$.

Since $w<w_{k}$ relative to deg-lex, it follows from the choice of $w_{k}$ that $w$ equals some regular group word modulo the relations of $\langle\mathfrak{A} \| \mathcal{S}\rangle$. Let $u$ be such a regular group word. By the choice of $w$,

$$
|u|>(n+1)|w|+n^{4} L .
$$

Let $\Delta$ be a weakly reduced special disc S-diagram over $\langle\mathfrak{A} \| \mathcal{S}\rangle$ such that the label of some representative of $\partial \Delta$ is $u w^{-1}$. Let $b$ and $p$ be the paths such that $\ell(b)=u, \ell(p)=w^{-1}$, and $\langle b p\rangle=\partial \Delta$. Let $b_{1}, b_{2}, \ldots, b_{n}$ be the subpaths of $b$ such that $b=b_{1} b_{2} \cdots b_{n}$, and for every $i=1,2, \ldots, n$, the label of $b_{i}$ is a power of $x_{i}$. For every $i=1,2, \ldots, n$, let $B_{i}$ be the set of all edges that lie on $b_{i}$. Let $\Sigma$ be the sum of the degrees of all the faces of $\Delta$. Let $T$ be the number of selected external edges of $\Delta$ that lie on $b$.

Since $\ell(b)$ is regular, no two oriented edges of $b$ are inverse to each other. Hence,

$$
|b| \leq \Sigma+|p|
$$


Let $\Sigma_{1}$ be the sum of the degrees of all faces of $\Delta$ that are incident with edges from at least 3 distinct sets from among $B_{1}, \ldots, B_{n}$. If $1 \leq i_{1}<i_{2}<i_{3} \leq n$, then there is at most one face that is incident to edges from all three sets $B_{i_{1}}, B_{i_{2}}, B_{i_{3}}$. Therefore, $\Sigma_{1} \leq n^{3} L$ (the degree of every face of $\Delta$ is at most $L$ ). Let $\Sigma_{2}$ be the sum of the degrees of all faces of $\Delta$ that are incident with edges from no more than 2 distinct sets from among $B_{1}, \ldots, B_{n}$.

One one hand,

$$
T \leq \Sigma_{1}+\frac{2}{n} \Sigma_{2} \leq n^{3} L+\frac{2}{n} \Sigma .
$$

On the other hand, by Proposition 5.1 and the Main Lemma,

$$
T+|p| \geq(1-2 \mu) \Sigma \text {. }
$$

Therefore,

$$
\begin{aligned}
(1-2 \mu) \Sigma & \leq n^{3} L+\frac{2}{n} \Sigma+|p|, \\
\left(1-2 \mu-\frac{2}{n}\right) \Sigma & \leq|p|+n^{3} L, \\
\frac{n-23}{n} \Sigma & \leq|p|+n^{3} L, \\
\Sigma & \leq n|p|+n^{4} L, \\
|b| & \leq(n+1)|p|+n^{4} L .
\end{aligned}
$$

In other terms, $|u| \leq(n+1)|w|+n^{4} L$. This gives a contradiction.

Thus, $w=w_{k}$, and the inductive step is done.

Everything is now ready to start proving properties of $G$.

Property 1. For every $(n-21)$-element subset I of the set $\{1, \ldots, n\}$, the system $\left\{a_{i}\right\}_{i \in I}$ freely generates a free subgroup $F$ of $G$ (of rank $\left.n-21\right)$. Moreover, every two elements in such a free subgroup $F$ are conjugate in $G$ only if they are conjugate in $F$.

Proof. Suppose that Property 1 does not hold. Take a set $I \subset\{1, \ldots, n\}$ of $n-21$ elements that provides a counterexample. Let $\mathfrak{B}=\left\{x_{i} \mid i \in I\right\}$.

If the system $\left\{a_{i}\right\}_{i \in I}$ does not freely generate a free subgroup of $G$, then there is a weakly reduced disc diagram $\Delta$ over $\langle\mathfrak{A} \| \mathcal{R}\rangle$ such that the label of some (therefore every) representative of its contour is a cyclically reduced nonempty group word over the alphabet $\mathfrak{B}$. Clearly, such a diagram $\Delta$ is non-degenerate (has a face).

If the system $\left\{a_{i}\right\}_{i \in I}$ does freely generate a free subgroup $F$ of $G$, but there are two elements of $F$ that are conjugate in $G$ but not in $F$, then let $v_{1}$ and $v_{2}$ be two cyclically reduced group words over $\mathfrak{B}$ that represent two such elements. The group words $v_{1}$ and $v_{2}$ are not cyclic shifts of each other, and neither of them represents the identity of $G$. Since $\left[v_{1}\right]$ and $\left[v_{2}\right]$ are nontrivial but are conjugate in $G$, there exists a weakly reduced annular diagram $\Delta$ over $\langle\mathfrak{A} \| \mathcal{R}\rangle$ such that the label of some representative of one of its contours is $v_{1}$, and the label of some representative of its other contour is $v_{2}^{-1}$ (see Lemma V.5.2 in LyS]). Clearly, such $\Delta$ is non-degenerate.

Thus, to obtain a contradiction and complete the proof, it is enough to show that there is no weakly reduced non-degenerate disc nor annular diagram over $\langle\mathfrak{A} \| \mathcal{R}\rangle$ with fewer than $n-20$ distinct basic letters on its contour(s). 
Let $\Delta$ be an arbitrary special weakly reduced non-degenerate disc or annular S-diagram over $\langle\mathfrak{A} \| \mathcal{R}\rangle$. (Recall that a special selection exists on every diagram over $\langle\mathfrak{A} \| \mathcal{R}\rangle$.) Suppose that $\Delta$ has fewer than $n-20$ distinct basic letters of $\mathfrak{A}$ on its contour(s). Let $S$ be the total number of selected external edges of $\Delta$. On one hand, by Proposition 5.2,

$$
S<\frac{n-21}{n} \sum_{\Pi \in \Delta(2)}|\partial \Pi| .
$$

On the other hand, by Corollary 1 of Proposition 5.1,

$$
S \geq(1-2 \mu) \sum_{\Pi \in \Delta(2)}|\partial \Pi| .
$$

Since

$$
1-2 \mu>\frac{n-21}{n}
$$

one has a contradiction.

The proof of the next property is not so straightforward.

Property 2. For every element $g$ of $G$, there exist unique $k_{1}, \ldots, k_{n} \in \mathbb{Z}$ such that $g=a_{1}^{k_{1}} \cdots a_{n}^{k_{n}}$.

The uniqueness is the "hard part" of Property 2. It shall be proved by contradiction. Main steps of the proof are stated below as Lemmas 5.1-5.16. These lemmas share some common assumptions about an S-diagram $\Delta$.

Assume that $\Delta$ is a special disc S-diagram over $\langle\mathfrak{A} \| \mathcal{R}\rangle$ whose contour has a representative of the form $p_{1} p_{2}$ such that $\ell\left(p_{1}\right)$ and $\ell\left(p_{2}^{-1}\right)$ are distinct regular group words, and $\ell\left(p_{1} p_{2}\right)$ is cyclically reduced. Suppose, moreover, that $\Delta$ is such an S-diagram with the minimal possible number of faces. In particular, if $\Delta^{\prime}$ is a nontrivial disc diagram over $\langle\mathfrak{A} \| \mathcal{R}\rangle, p$ is a representative of $\partial \Delta^{\prime}$, and $\ell(p)$ is regular or counter-regular, then $\Delta^{\prime}$ has no fewer faces than $\Delta$.

Lemma 5.1. The diagram $\Delta$ is weakly reduced.

Proof. This easily follows from the minimality of the number of faces of $\Delta$.

Lemma 5.2. The diagram $\Delta$ is simple.

Proof. Use the minimality of $\Delta$ again. Some maximal simple disc S-subdiagram of $\Delta$ satisfies all the assumptions made about $\Delta$ (this follows, for example, from Proposition 3.1 of $[\mathrm{Mu}]$ ), and hence it must be the whole of $\Delta$.

Lemma 5.3. If $\Delta^{\prime}$ is a disc $S$-subdiagram of $\Delta$, and $S$ is the number of selected external edge of $\Delta^{\prime}$ (external in $\left.\Delta^{\prime}\right)$, then

$$
S \geq(1-2 \mu) \sum_{\Pi \in \Delta^{\prime}(2)}|\partial \Pi| .
$$

Proof. Follows directly from Lemma 5.1 and Corollary 1 of Proposition 5.1.

Lemma 5.4. If $\Delta^{\prime}$ is a disc subdiagram of $\Delta, p_{1}$ and $p_{2}$ are paths, $\left\langle p_{1} p_{2}\right\rangle=\partial \Delta^{\prime}$, and the reduced forms of $\ell\left(p_{1}\right)$ and $\ell\left(p_{2}^{-1}\right)$ are regular, then either $\Delta^{\prime}$ is degenerate, or it coincides with $\Delta$. 


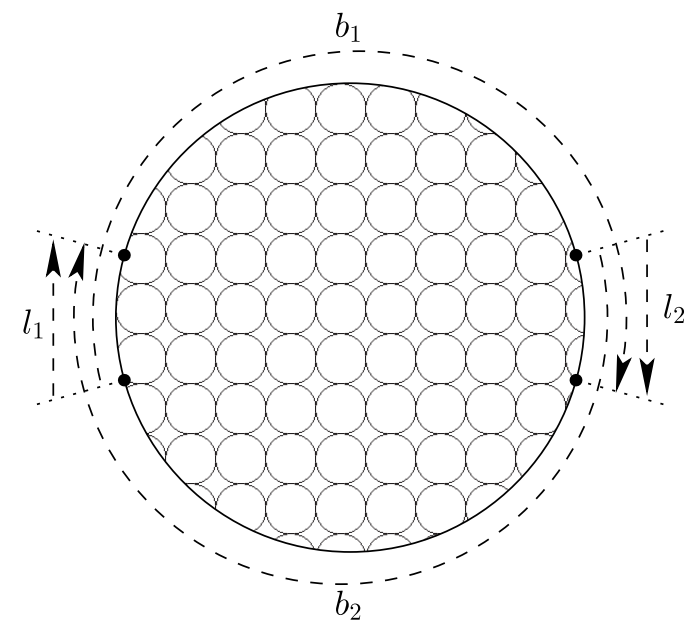

Figure 1 . The paths $b_{1}, b_{2}, l_{1}$, and $l_{2}$ in $\Delta$.

Proof. Suppose $\Delta^{\prime}, p_{1}$, and $p_{2}$ are such as in the hypotheses of the lemma.

Case 1: $\ell\left(p_{1}\right)$ and $\ell\left(p_{2}^{-1}\right)$ are freely equal. Then the label of every representative of $\partial \Delta^{\prime}$ is freely trivial and therefore $\Delta^{\prime}$ is degenerate. Indeed, if $\Delta^{\prime}$ was nondegenerate, there would exist a disc diagram over $\langle\mathfrak{A} \| \mathcal{R}\rangle$ with the same label of the contour as $\Delta$ but with fewer faces (faces of $\Delta^{\prime}$, possibly together with some of the others, could be "eliminated" from $\Delta$ ), which would contradict the minimality of $\Delta$.

Case 2: $\ell\left(p_{1}\right)$ and $\ell\left(p_{2}^{-1}\right)$ are not freely equal. Then take $\Delta^{\prime}$ and repeatedly fold labelled external edges and "cut off," whenever necessary, "branches" with freely trivial contour labels until one obtains a disc diagram $\Delta^{\prime \prime}$ such that the labels of the representatives of $\partial \Delta^{\prime \prime}$ are cyclically reduced. Clearly, $\Delta^{\prime \prime}$ is a nondegenerate diagram over $\langle\mathfrak{A} \| \mathcal{R}\rangle$, the label of every representative of $\partial \Delta^{\prime \prime}$ is a cyclically reduced form of $\ell\left(p_{1} p_{2}\right)$, and $\left\|\Delta^{\prime \prime}(2)\right\| \leq\left\|\Delta^{\prime}(2)\right\|$. By the minimality of $\Delta$, such a diagram $\Delta^{\prime \prime}$ cannot have fewer faces than $\Delta$. Therefore, $\Delta^{\prime}(2)=\Delta(2)$ and, since $\partial \Delta$ is reduced, $\Delta^{\prime}$ coincides with $\Delta$.

Lemma 5.5. The diagram $\Delta$ has more than 1 face.

Proof. This easily follows from the form of elements of $\mathcal{R}$.

Lemma 5.6. There are at least $n-20$ distinct basic letters on $\partial \Delta$.

Proof. Follows from Property 1.

Let $b_{1}$ be the maximal subpath of $\partial \Delta$ whose label is regular but not counterregular (is not a letter power). Let $b_{2}$ be the maximal subpath of $\partial \Delta$ whose label is counter-regular but not regular. Since, according to Lemma 5.6, there are at least 2 distinct basic letters on $\partial \Delta$, it follows that the paths $b_{1}$ and $b_{2}$ are well-defined. (If, say, the label of some representative of $\partial \Delta$ is $x_{1} x_{2} x_{3} \cdots x_{42} x_{43} x_{42} x_{2} x_{1}$, then $\ell\left(b_{1}\right)=x_{1}^{2} x_{2} \cdots x_{43}$, and $\ell\left(b_{2}\right)=x_{43} x_{42} x_{2} x_{1}^{2}$.) The label of each of the paths $b_{1}$ and $b_{2}$ has at least 2 distinct basic letters. Let $l_{1}$ be the initial subpath of $b_{1}$ that is a terminal subpath of $b_{2}$, and let $l_{2}$ be the initial subpath of $b_{2}$ that is a terminal 
subpath of $b_{1}$. Both $l_{1}$ and $l_{2}$ are nontrivial (see Figure 1 ). The labels of $l_{1}$ and $l_{2}$ are letter powers.

Lemma 5.7. Every selected external arc of $\Delta$ lies on at least one of the paths $b_{1}$ or $b_{2}$.

Proof. Follows from the fact that the label of every selected oriented arc is either regular or counter-regular.

The following observation is obvious but deserves mentioning because it is used implicitly in the proofs of Lemmas 5.8, 5.10, and 5.13 several times.

Suppose $p_{1}, p_{2}$, and $q$ are paths in a diagram over $\langle\mathfrak{A} \| \mathcal{R}\rangle$, and $q$ is nontrivial. Then

- if the products $p_{1} q$ and $q^{-1} p_{2}$ are defined and their labels are regular, then $\ell(q)$ is a letter power, and the reduced form of $\ell\left(p_{1} p_{2}\right)$ is regular;

- if the products $p_{1} q$ and $q p_{2}$ are defined and their labels are regular, then $\ell\left(p_{1} q p_{2}\right)$ is regular.

Since Lemma 5.7 and many of the remaining lemmas deal with selected external arcs or selected external oriented arcs, it is advisable to review these concepts. An oriented arc of a graph (or a 2-complex) is a simple path all of whose vertices, except, possibly, the end-vertices, have degree two in the given graph. A (nonoriented) arc is a pair of mutually inverse oriented arcs. An arc is incident to a face $\Pi$ if (and only if) at least one of the associated oriented arcs is a subpath of $\partial \Pi$. Every external arc of a (semi-)simple map is incident to exactly one face. An oriented arc is selected if (and only if) it is selected as a path (in particular, it must be a subpath of the contour of some face). An external arc is selected if (and only if) it is incident to some face $\Pi$, and the associated oriented arc that is a subpath of $\partial \Pi$ is selected. Distinct maximal selected external arcs of $\Delta$ never overlap.

Lemma 5.8. In the diagram $\Delta$, every face is incident with at most 1 maximal selected external arc.

Proof. Consider an arbitrary face $\Pi$ of $\Delta$. Suppose $\Pi$ is incident with at least 2 distinct maximal selected external arcs.

Let $u_{1}$ and $u_{2}$ be distinct maximal selected external oriented arcs of $\Delta$ that are subpaths of $\partial \Pi$. (They do not overlap.) Let $s$ be the maximal selected subpath of $\partial \Pi$. Without loss of generality, assume that $u_{1}$ precedes $u_{2}$ as a subpath of $s$. Let $v$ be such a path that $u_{1} v u_{2}$ is a subpath of $s$. Since $\Delta$ is simple (see Lemma 5.2), and $u_{1}$ and $u_{2}$ are maximal, it follows that $v$ is nontrivial, and the first and last oriented edges of $v$ are internal in $\Delta$.

Suppose $v$ has a simple cyclic subpath $p$. Then $\langle p\rangle$ is the contour of a proper simple disc subdiagram of $\Delta$. Since $\ell(p)$ is regular or counter-regular, this contradicts the minimality of $\Delta$. Hence, the path $v$ is simple and not cyclic (equivalently, every vertex occurs in $v$ at most once).

Let $v_{1}$ be the minimal subpath of $\partial \Delta$ such that $u_{1}^{-1}$ and $u_{2}^{-1}$ are respectively its initial and terminal subpaths. Let $v_{2}$ be the path such that $\left\langle v_{1} v_{2}\right\rangle=\partial \Delta$. The paths $v_{1} v^{-1}$ and $v_{2} v$ are cyclically reduced and represent the contours of two disc subdiagrams of $\Delta$. Let $\Delta_{1}^{\prime}$ and $\Delta_{2}^{\prime}$ be the subdiagrams with the contours $\left\langle v_{1} v^{-1}\right\rangle$ and $\left\langle v_{2} v\right\rangle$, respectively. Note that $\Pi \in \Delta_{1}^{\prime}(2)$, and $\Delta(2)$ is the disjoint union of 


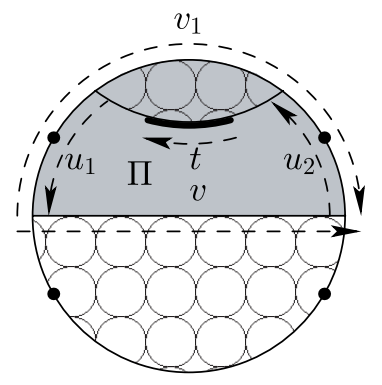

Case 1
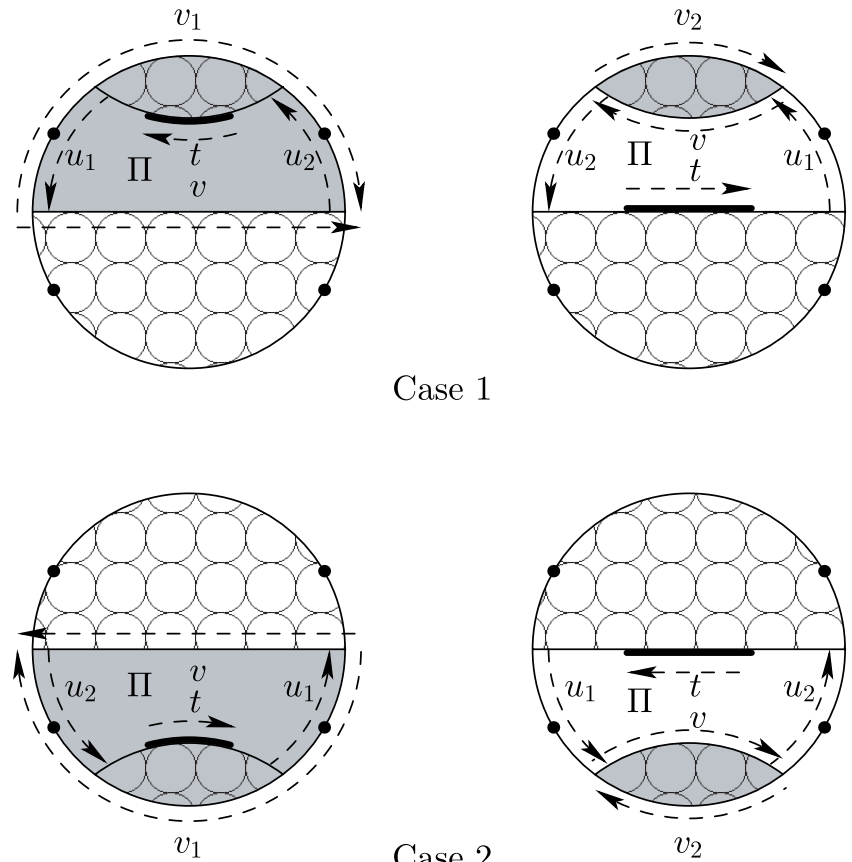

Case 2

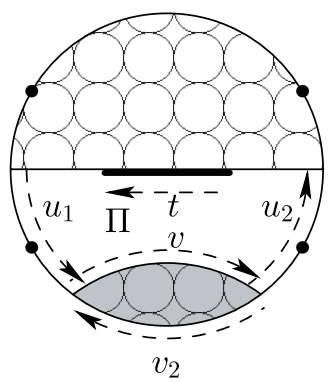

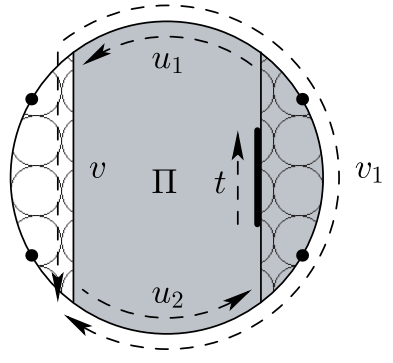

Case 3

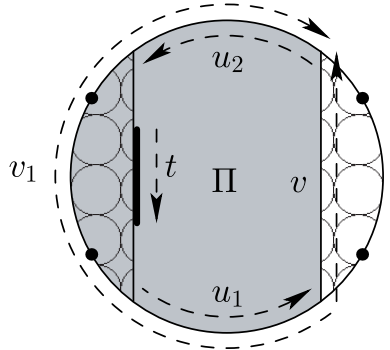

Case 4

Figure 2. Cases 1-4, Lemma 5.8.

$\Delta_{1}^{\prime}(2)$ and $\Delta_{2}^{\prime}(2)$. The diagram $\Delta_{1}^{\prime}$ is simple. The diagram $\Delta_{2}^{\prime}$ is non-degenerate. Both $\Delta_{1}^{\prime}$ and $\Delta_{2}^{\prime}$ are proper subdiagrams of $\Delta$.

The labels of $u_{1}$ and $u_{2}$ are both regular or both counter-regular. Therefore, each of the paths $u_{1}^{-1}$ and $u_{2}^{-1}$ is a subpath of one of the paths $b_{1}$ or $b_{2}$ (see Lemma 5.7). Consider the following 4 cases (see Figure 2; shaded subdiagrams are the "sources of contradiction"):

Case 1: $u_{1}^{-1}$ and $u_{2}^{-1}$ are subpaths of $b_{1}$. Then either $v_{1}$ or $u_{2}^{-1} v_{2} u_{1}^{-1}$ is a subpath of $b_{1}$ as well. Suppose $v_{1}$ is a subpath of $b_{1}$. Then $\ell\left(v_{1}\right)$ is regular, $\ell\left(u_{1}\right)$ and $\ell\left(u_{2}\right)$ are letter powers, and $\ell\left(u_{1} v u_{2}\right)$ is regular. Therefore, $\left\langle v_{1} v^{-1}\right\rangle$ cannot be the contour of a proper non-degenerate disc subdiagram (see Lemma 5.4). This gives a contradiction. Suppose $u_{2}^{-1} v_{2} u_{1}^{-1}$ is a subpath of $b_{1}$. Then $\ell\left(u_{2}^{-1} v_{2} u_{1}^{-1}\right)$ is 
regular, and $\ell\left(u_{1} v u_{2}\right)$ is counter-regular. Therefore, $\left\langle v_{2} v\right\rangle$ cannot be the contour of a proper non-degenerate disc subdiagram. This gives a contradiction.

Case 2: $u_{1}^{-1}$ and $u_{2}^{-1}$ are subpaths of $b_{2}$. When proving impossibility of Case 1 , it was essentially shown that no face of $\Delta$ can be incident with 2 distinct maximal selected external arcs lying on $b_{1}$. Since all the assumptions made about $\Delta$ hold for its mirror copy as well, the same statement appropriately reformulated must hold for the mirror copy of $\Delta$. Namely, no face of the mirror copy of $\Delta$ can be incident with 2 distinct maximal selected external arcs lying on $b_{2}^{-1}\left(b_{2}^{-1}\right.$ plays the same role for the mirror copy of $\Delta$ as $b_{1}$ does for $\Delta$ ). This means that Case 2 is impossible.

Case 3: $u_{1}^{-1}$ is a subpath of $b_{1}$, and $u_{2}^{-1}$ is a subpath of $b_{2}$. Pick a subpath $p_{1}$ of $b_{1}$ and a subpath $p_{2}$ of $b_{2}$ such that $u_{1}^{-1}$ is an initial subpath of $p_{1}, u_{2}^{-1}$ is a terminal subpath of $p_{2}$, and $p_{1} p_{2}=v_{1}$. Then $\ell\left(p_{1}\right)$ is regular, $\ell\left(p_{2}\right)$ is counter-regular, and $\ell\left(u_{2}^{-1} v^{-1} u_{1}^{-1}\right)$ is regular or counter-regular. If $\ell\left(u_{2}^{-1} v^{-1} u_{1}^{-1}\right)$ is regular, then $\ell\left(v^{-1} p_{1}\right)$ is regular. If $\ell\left(u_{2}^{-1} v^{-1} u_{1}^{-1}\right)$ is counter-regular, then $\ell\left(p_{2} v^{-1}\right)$ is counterregular. In either case $\left\langle p_{2} v^{-1} p_{1}\right\rangle=\left\langle v_{1} v^{-1}\right\rangle$ cannot be the contour of a proper non-degenerate disc subdiagram. This gives a contradiction.

Case 4: $u_{1}^{-1}$ is a subpath of $b_{2}$, and $u_{2}^{-1}$ is a subpath of $b_{1}$. Pick a subpath $p_{1}$ of $b_{2}$ and a subpath $p_{2}$ of $b_{1}$ such that $u_{1}^{-1}$ is an initial subpath of $p_{1}, u_{2}^{-1}$ is a terminal subpath of $p_{2}$, and $p_{1} p_{2}=v_{1}$. Then $\ell\left(p_{1}\right)$ is counter-regular, $\ell\left(p_{2}\right)$ is regular, and $\ell\left(u_{2}^{-1} v^{-1} u_{1}^{-1}\right)$ is regular or counter-regular. If $\ell\left(u_{2}^{-1} v^{-1} u_{1}^{-1}\right)$ is regular, then $\ell\left(p_{2} v^{-1}\right)$ is regular. If $\ell\left(u_{2}^{-1} v^{-1} u_{1}^{-1}\right)$ is counter-regular, then $\ell\left(v^{-1} p_{1}\right)$ is counterregular. In either case $\left\langle p_{2} v^{-1} p_{1}\right\rangle=\left\langle v_{1} v^{-1}\right\rangle$ cannot be the contour of a proper nondegenerate disc subdiagram. This gives a contradiction.

(Case 4 is symmetric with Case 3 , but this symmetry is not simply the mirror symmetry as between Cases 1 and 2. Say, if Case 4 was indeed the case for $\Delta$, then the mirror copy of $\Delta$ would be just another example for the same Case 4 , not Case 3.)

A contradiction is obtained in each of the 4 cases, and no other cases exist.

Lemma 5.9. There are a face $\Pi$ and a path $s^{\prime}$ in $\Delta$ such that:

- $s^{\prime}$ is a selected subpath of $\partial \Pi$;

- $s^{\prime}$ is a maximal selected external oriented arcs of $\Delta$;

- the $n-40$ basic letters $x_{21}, x_{22}, \ldots, x_{n-20}$ all occur in $\ell\left(s^{\prime}\right)$.

Proof. For every face $\Pi$ of $\Delta$, let $S(\Pi)$ denote the number of selected external edges of $\Delta$ incident to $\Pi$. By Lemma 5.3,

$$
\sum_{\Pi \in \Delta(2)} S(\Pi) \geq(1-2 \mu) \sum_{\Pi \in \Delta(2)}|\partial \Pi| .
$$

Hence, there exists a face $\Pi$ of $\Delta$ such that

$$
S(\Pi) \geq(1-2 \mu)|\partial \Pi|>\frac{n-21}{n}|\partial \Pi|
$$

(recall that $1-2 \mu>1-21 / n$ ).

Let $\Pi$ be such a face as above. Let $s$ be the maximal selected subpath of $\partial \Pi$. Let $s^{\prime}$ be the (only) maximal selected external oriented arc of $\Delta$ that is a subpath of $s$ (see Lemma 5.8). Then

$$
\left|s^{\prime}\right|=S(\Pi)>\frac{n-21}{n}|\partial \Pi|>\frac{n-21}{n}|s| .
$$


Let $j=\operatorname{rank}(\Pi)$. Then either $\ell(s)=x_{1}^{m_{j}} x_{2}^{m_{j}} \cdots x_{n}^{m_{j}}$ or $\ell(s)=x_{n}^{-m_{j}} x_{n-1}^{-m_{j}} \cdots x_{1}^{-m_{j}}$. Therefore, $\ell\left(s^{\prime}\right)$ has at least $n-20$ distinct basic letters, and all of the basic letters $x_{21}, x_{22}, \ldots, x_{n-20}$ occur in it.

Lemmas 5.1-5.9, as well as 5.10-5.16, assert some properties of $\Delta$. Observe that none of these properties in fact can distinguish between $\Delta$ and its mirror copy, i.e., each of these properties holds for $\Delta$ if and only if it holds for the mirror copy of $\Delta$. Thus, at this point analogues of Lemmas 5.1-5.9 for the mirror copy of $\Delta$ shall be assumed proved. Moreover, the initial assumptions about the S-diagram $\Delta$ are also true about its mirror copy.

Even though the statement of Lemma 5.7 is about the S-diagram $\Delta$ and the paths $b_{1}$ and $b_{2}$, it still may be viewed as an assertion of a property of $\Delta$ because, according to the way they are chosen, $b_{1}$ and $b_{2}$ are uniquely determined for the given $\Delta$. Therefore, the analog of Lemma 5.7 for the mirror copy of $\Delta$ states:

Every selected external arc of the mirror copy of $\Delta$ lies on at least one of the paths $b_{2}^{-1}$ or $b_{1}^{-1}$.

In the proofs of Lemmas $5.10-5.13$, it is convenient in some cases to pass to the mirror copy of $\Delta$ to reduce the number of cases to consider.

Lemma 5.10. Let $\Pi_{1}$ be a face of $\Delta$ incident to a selected external edge of $\Delta$, and let $\Pi_{2}$ be another face of $\Delta$. Let $s_{1}$ and $s_{2}$ be the maximal selected subpaths of $\partial \Pi_{1}$ and $\partial \Pi_{2}$, respectively. Let $s_{1}^{\prime}$ be the maximal selected external oriented arc of $\Delta$ that is a subpath of $s_{1}$. Let $s_{1-}^{\prime}$ and $s_{1+}^{\prime}$ be the paths such that $s_{1}=s_{1-}^{\prime} s_{1}^{\prime} s_{1+}^{\prime}$. Let $q_{1}$ be the path such that $\left\langle s_{1}^{\prime-1} q_{1}\right\rangle=\partial \Delta$. Suppose there are at least 2 distinct basic letters in $\ell\left(s_{1}^{\prime}\right)$. Suppose the paths $s_{2}$ and $\left(s_{1-}^{\prime} q_{1} s_{1+}^{\prime}\right)^{-1}$ have a common oriented edge. Then they have exactly one maximal common nontrivial subpath.

Proof. Observe that to prove that there is exactly one maximal common nontrivial subpath of $s_{2}$ and $\left(s_{1-}^{\prime} q_{1} s_{1+}^{\prime}\right)^{-1}$, it suffices to prove the same for $s_{2}^{-1}$ and $s_{1-}^{\prime} q_{1} s_{1+}^{\prime}$. This justifies passing to the mirror copy of $\Delta$.

Since the paths $s_{2}$ and $\left(s_{1-}^{\prime} q_{1} s_{1+}^{\prime}\right)^{-1}$ have a common oriented edge, they have at least one maximal common nontrivial subpath.

The label of $s_{1}^{\prime}$ is regular or counter-regular. Therefore, $s_{1}^{\prime}$ is a subpath of $b_{1}^{-1}$ or $b_{2}^{-1}$. If it is a subpath of $b_{2}^{-1}$ but not of $b_{1}^{-1}$, pass from $\Delta, b_{1}, b_{2}, l_{1}, l_{2}, s_{1}, s_{2}$, $s_{1-}^{\prime}, s_{1+}^{\prime}, s_{1}^{\prime}, q_{1}$ to the mirror copy of $\Delta, b_{2}^{-1}, b_{1}^{-1}, l_{1}^{-1}, l_{2}^{-1}, s_{1}^{-1}, s_{2}^{-1}, s_{1+}^{\prime-1}, s_{1-}^{\prime-1}$, $s_{1}^{\prime-1}, q_{1}^{-1}$, respectively. Hence, it may and shall be assumed that $s_{1}^{\prime}$ is a subpath of $b_{1}^{-1}$ (see Figure 3$)$.

Let $t_{1}$ be the path such that $\left\langle s_{1} t_{1}\right\rangle=\partial \Pi_{1}$. Let $y_{-}$and $y_{+}$be the paths such that $b_{1}=y_{-} s_{1}^{\prime-1} y_{+}$. Let $b_{2}^{\prime}$ be the subpath of $b_{2}$ such that $\left\langle b_{1} b_{2}^{\prime}\right\rangle=\partial \Delta$. Then $b_{2}=l_{2} b_{2}^{\prime} l_{1}$ and $q_{1}=y_{+} b_{2}^{\prime} y_{-}$.

Since $\ell\left(b_{1}^{-1}\right)$ is counter-regular, and $\ell\left(s_{1}^{\prime}\right)$ is not a letter power (it has at least 2 distinct basic letters $), \ell\left(s_{1}\right)$ is counter-regular as well. Hence, $\ell\left(s_{1+}^{\prime-1} s_{1}^{\prime-1} y_{+}\right)$and $\ell\left(y_{-} s_{1}^{\prime-1} s_{1-}^{\prime-1}\right)$ are regular.

Let $j_{1}=\operatorname{rank}\left(\Pi_{1}\right)$. Then $\ell\left(s_{1}\right)=x_{n}^{-m_{j_{1}}} x_{n-1}^{-m_{j_{1}}} \cdots x_{1}^{-m_{j_{1}}}$ and $\ell\left(t_{1}\right)=w_{j_{1}}$.

Since $\ell\left(s_{1}^{\prime}\right)$ has at least 2 distinct basic letters, $\ell\left(y_{-} s_{1+}^{\prime}\right)$ and $\ell\left(s_{1-}^{\prime} y_{+}\right)$have disjoint sets of basic letters. This also implies that $l_{1}$ is a proper initial subpath of $y_{-} s_{1}^{\prime-1}$, and $l_{2}$ is a proper terminal subpath of $s_{1}^{\prime-1} y_{+}$. 


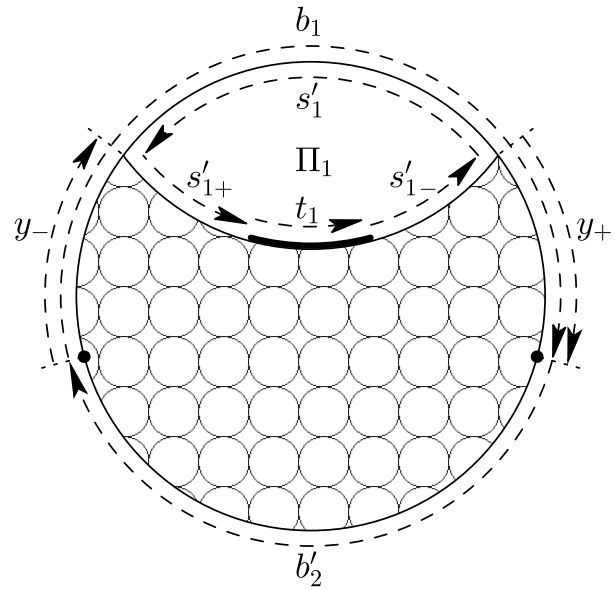

Figure 3 . The face $\Pi_{1}$ in $\Delta$, Lemma 5.10 .

Suppose $s_{2}$ and $\left(s_{1-}^{\prime} q_{1} s_{1+}^{\prime}\right)^{-1}$ have at least two distinct maximal common nontrivial subpaths. Then let $u_{1}$ and $u_{2}$ be two paths such that:

- $u_{1}$ and $u_{2}$ are nontrivial subpaths of distinct maximal common subpaths of $s_{2}$ and $\left(s_{1-}^{\prime} q_{1} s_{1+}^{\prime}\right)^{-1}$,

- $u_{1}$ precedes $u_{2}$ as a subpath of $s_{2}$, and

- each of the paths $u_{1}$ and $u_{2}$ is a maximal common subpath of $s_{2}$ and one of the paths $y_{-}^{-1}, y_{+}^{-1}, s_{1-}^{\prime-1}, s_{1+}^{\prime-1}$, or $b_{2}^{\prime-1}$.

Clearly, such $u_{1}$ and $u_{2}$ exist and are nonoverlapping oriented arcs of $\Delta$. Let $v$ be the path such that $u_{1} v u_{2}$ is a subpath of $s_{2}$. Since $u_{1}$ and $u_{2}$ are subpaths of distinct maximal common subpaths of $s_{2}$ and $\left(s_{1-}^{\prime} q_{1} s_{1+}^{\prime}\right)^{-1}$, the path $u_{1} v u_{2}$ is not a subpath of $\left(s_{1-}^{\prime} q_{1} s_{1+}^{\prime}\right)^{-1}$.

Consider the following 16 cases (see Figures 4-8):

(Notice that in a certain sense Cases $6,8,10,12,14$, and 16 are "symmetric" with Cases 5, 7, 9, 11, 13, and 15, respectively.)

Case 1: each of the paths $u_{1}$ and $u_{2}$ is a subpath of $q_{1}^{-1}$. Then $u_{1}$ and $u_{2}$ are external selected oriented arcs of $\Delta$. Since there is only one maximal selected external arc of $\Delta$ incident to $\Pi_{2}$ (see Lemma 5.8), the path $u_{1} v u_{2}$ is a subpath of $q_{1}^{-1}$. This gives a contradiction.

Case 2: both $u_{1}$ and $u_{2}$ are subpaths of one of the paths $s_{1-}^{\prime-1}$ or $s_{1+}^{\prime-1}$. Let $z$ be one of the paths $s_{1-}^{\prime}$ or $s_{1+}^{\prime}$ such that both $u_{1}$ and $u_{2}$ are subpaths of $z^{-1}$. Then $\ell(z)$ is counter-regular. Suppose $u_{2}$ precedes $u_{1}$ as a subpath of $z^{-1}$. Let $p$ be the path such that $u_{1}^{-1} p u_{2}^{-1}$ is a subpath of $z$. Then $\ell\left(u_{1}^{-1} p u_{2}^{-1}\right)$ is counter-regular. Therefore, $\ell\left(u_{2}^{-1} v^{-1} u_{1}^{-1}\right)$ is regular. The cycle $\left\langle p u_{2}^{-1} v^{-1} u_{1}^{-1}\right\rangle$ is the contour of a disc subdiagram of $\Delta$ not containing the face $\Pi_{1}$ but containing the face $\Pi_{2}$. This contradicts the minimality of $\Delta$. Therefore, $u_{1}$ precedes $u_{2}$ as a subpath of $z^{-1}$. Let $p$ be the path such that $u_{2}^{-1} p u_{1}^{-1}$ is a subpath of $z$. Then $\ell\left(u_{2}^{-1} p u_{1}^{-1}\right)$ is counter-regular. Therefore, $\ell\left(u_{1} v u_{2}\right)$ is regular. The cycle $\langle p v\rangle$ is the contour of a disc subdiagram of $\Delta$ not containing the faces $\Pi_{1}$ and $\Pi_{2}$. By Lemma 5.4 (by the minimality of $\Delta$ ), this subdiagram is degenerate. Since $z$ and $v$ are reduced, $v=p^{-1}$. Therefore, $u_{1} v u_{2}$ is a subpath of $z^{-1}$. This gives a contradiction. 

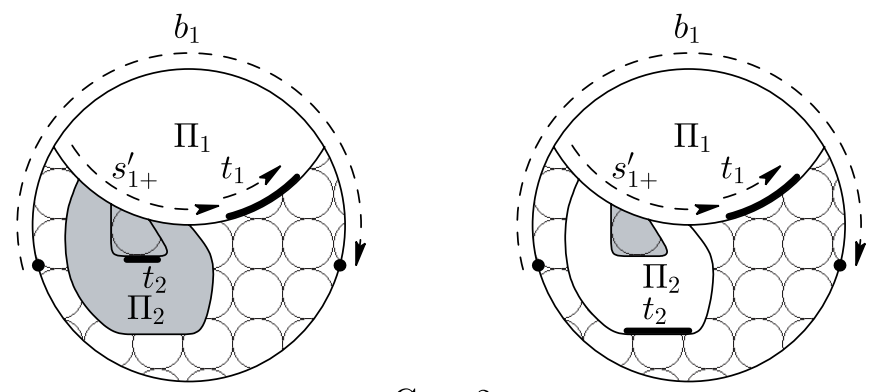

Case 2

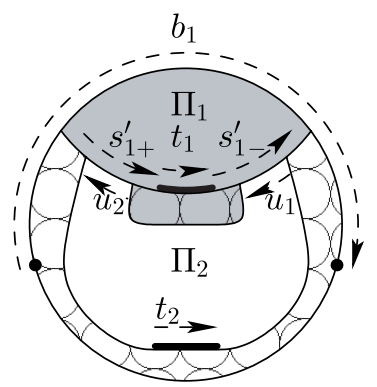

Case 3

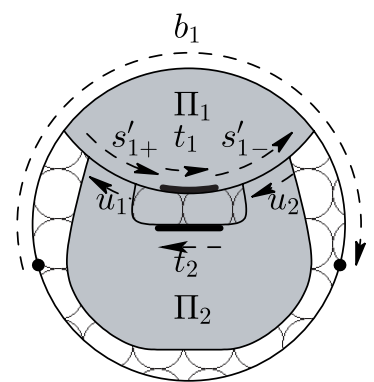

Case 4

Figure 4. Cases 2, 3, 4, Lemma 5.10.

Case 3: $u_{1}$ is a subpath of $s_{1-}^{\prime-1}$, and $u_{2}$ is a subpath of $s_{1+}^{\prime-1}$. Let $p$ be the path such that $u_{1}^{-1} p^{-1} u_{2}^{-1}$ is a subpath of $s_{1}$. Then $\ell\left(u_{2} p u_{1}\right)$ is regular. Therefore, $\ell\left(u_{1} v u_{2}\right)$ is counter-regular. The cycle $\left\langle p u_{1} v u_{2}\right\rangle$ is the contour of a disc subdiagram of $\Delta$ containing the face $\Pi_{1}$ but not containing the face $\Pi_{2}$. This contradicts the minimality of $\Delta$ (see Lemma 5.4).

Case 4: $u_{1}$ is a subpath of $s_{1+}^{\prime-1}$, and $u_{2}$ is a subpath of $s_{1-}^{\prime-1}$. Since the labels of $s_{1-}^{\prime}$ and $s_{1+}^{\prime}$ have disjoint sets of basic letters, $\ell\left(s_{2}\right)$ is regular. Let $p$ be the path such that $u_{2}^{-1} p^{-1} u_{1}^{-1}$ is a subpath of $s_{1}$. Then $\ell\left(u_{1} v u_{2}\right)$ and $\ell\left(u_{1} p u_{2}\right)$ are regular. If $v$ is trivial, then the cyclic path $p v^{-1}=p$ is cyclically reduced because $\ell(p)$ is regular. If $v$ is nontrivial, then the cyclic path $p v^{-1}$ is cyclically reduced by the maximality of $u_{1}$ and $u_{2}$, and because the pathes $v$ and $p$ are reduced. Suppose the path $p v^{-1}$ is not simple. Then some subpath of some cyclic shift of $v p^{-1}$ is a representative of the contour of a proper simple disc subdiagram of $\Delta$. Let $\Delta^{\prime}$ be such a subdiagram. At least one of the following three subcases takes place:

(a) there is a subpath of $v$ or $p^{-1}$ which represents $\partial \Delta^{\prime}$, or

(b) there are paths $v^{\prime}$ and $p^{\prime}$ such that $\left\langle v^{\prime} p^{\prime-1}\right\rangle=\partial \Delta^{\prime}$, and the paths $v^{\prime}$ and $p^{\prime}$ are either terminal subpaths of $v$ and $p$, respectively, or initial subpaths of $v$ and $p$, respectively, or

(c) there are an initial subpath $p_{-}^{\prime}$ and a terminal subpath $p_{+}^{\prime}$ of the path $p$ such that $\left\langle v p_{+}^{\prime-1} p_{-}^{\prime-1}\right\rangle=\partial \Delta^{\prime}$.

In subcase (c) note that $\ell\left(p_{-}^{\prime} p_{+}^{\prime}\right)$ is regular. Lemma 5.4 easily yields a contradiction in each subcase. Hence, the cyclic path $p v^{-1}$ is simple. Therefore, it represents 


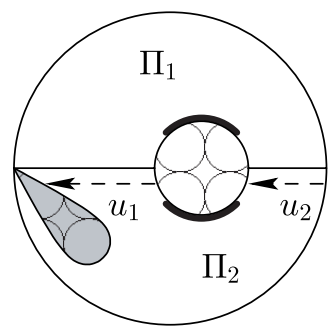

Case 4(a)

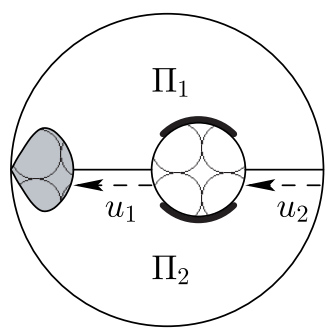

Case 4(b)
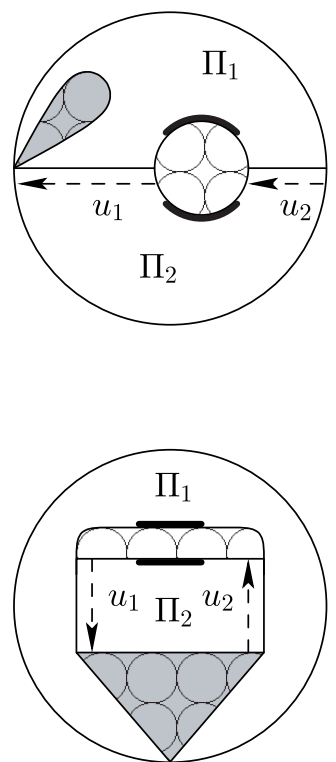

Case 4(c)

Figure 5. Special subcases of Case 4, Lemma 5.10.

the contour of a simple disc subdiagram of $\Delta$ containing the faces $\Pi_{1}$ and $\Pi_{2}$. By Lemma 5.4, $\left\langle p v^{-1}\right\rangle=\partial \Delta$. Therefore, $v=q_{1}^{-1}$, and $u_{1} v u_{2}$ is a subpath of $\left(s_{1-}^{\prime} q_{1} s_{1+}^{\prime}\right)^{-1}$. This gives a contradiction.

Case 5: $u_{1}$ is a subpath of $s_{1-}^{\prime-1}$, and $u_{2}$ is a subpath of $y_{-}^{-1}$. Let $p$ be the path such that $u_{2}^{-1} p u_{1}$ is a subpath of $y_{-} s_{1}^{\prime-1} s_{1-}^{\prime-1}$. Then $\ell\left(u_{2}^{-1} p u_{1}\right)$ is regular. Therefore, $\ell\left(u_{1} v u_{2}\right)$ is counter-regular. The cycle $\left\langle p u_{1} v\right\rangle$ is the contour of a disc subdiagram of $\Delta$ containing the face $\Pi_{1}$ but not containing the face $\Pi_{2}$. This contradicts the minimality of $\Delta$ (see Lemma 5.4).

Case 6: $u_{1}$ is a subpath of $y_{+}^{-1}$, and $u_{2}$ is a subpath of $s_{1+}^{\prime-1}$. Let $p$ be the path such that $u_{2} p u_{1}^{-1}$ is a subpath of $s_{1+}^{\prime-1} s_{1}^{\prime-1} y_{+}$. Then $\ell\left(u_{2} p u_{1}^{-1}\right)$ is regular. Therefore, $\ell\left(u_{1} v u_{2}\right)$ is counter-regular. The cycle $\left\langle p v u_{2}\right\rangle$ is the contour of a disc subdiagram of $\Delta$ containing the face $\Pi_{1}$ but not containing the face $\Pi_{2}$. This contradicts the minimality of $\Delta$.

Case 7: $u_{1}$ is a subpath of $y_{-}^{-1}$, and $u_{2}$ is a subpath of $s_{1-}^{\prime-1}$. Let $p$ be the path such that $u_{1}^{-1} p u_{2}$ is a subpath of $y_{-} s_{1}^{\prime-1} s_{1-}^{\prime-1}$. The cyclic path $p v^{-1} u_{1}^{-1}$ is cyclically reduced. Suppose it is not simple. Then at least one of the following two subcases takes place:

(a) there is a subpath of $v$ or $p^{-1}$ which represents the contour of a proper simple disc subdiagram of $\Delta$, or

(b) there are paths $v^{\prime}$ and $p^{\prime}$ such that $\left\langle v^{\prime} p^{-1}\right\rangle$ is the contour of a proper simple disc subdiagram of $\Delta$, and $v^{\prime}$ and $p^{\prime}$ are terminal subpaths of $v$ and $p$, respectively.

Lemma 5.4 yields a contradiction in both subcases. Hence, the cyclic path $p v^{-1} u_{1}^{-1}$ is simple. Therefore, it represents the contour of a disc subdiagram of $\Delta$ containing 


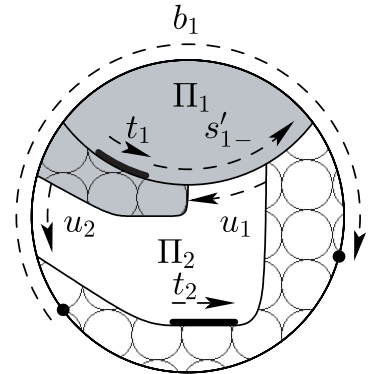

Case 5

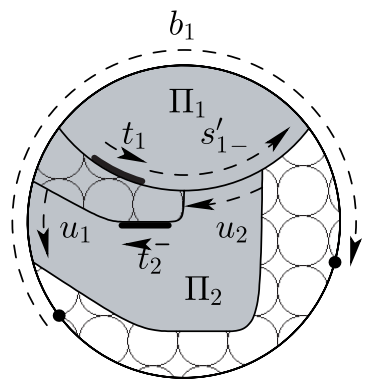

Case 7

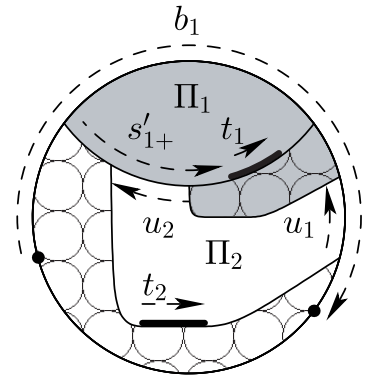

Case 6

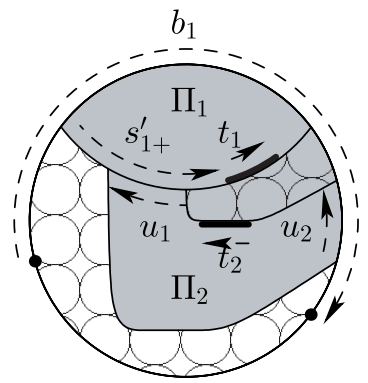

Case 8

Figure 6. Cases 5-8, Lemma 5.10.

the faces $\Pi_{1}$ and $\Pi_{2}$. The label of $u_{1}^{-1} p u_{2}$ is regular. Therefore, $\ell\left(u_{2}^{-1} v^{-1} u_{1}^{-1}\right)$ is counter-regular. By Lemma 5.4, $\left\langle p v^{-1} u_{1}^{-1}\right\rangle=\partial \Delta$. Therefore, $u_{1} v u_{2}$ is a subpath of $\left(s_{1-}^{\prime} q_{1}\right)^{-1}$. This gives a contradiction.

Case 8: $u_{1}$ is a subpath of $s_{1+}^{-1}$, and $u_{2}$ is a subpath of $y_{+}^{-1}$. Arguing as in Case 7, one obtains that $u_{1} v u_{2}$ is a subpath of $\left(q_{1} s_{1+}^{\prime}\right)^{-1}$. This gives a contradiction.

Case 9: $u_{1}$ is a subpath of $s_{1-}^{\prime-1}$, and $u_{2}$ is a subpath of $y_{+}^{-1}$. Let $p_{1}$ and $p_{2}$ be the paths such that $u_{1}^{-1} p_{1}$ is a terminal subpath of $s_{1-}^{\prime}$, and $p_{2} u_{2}^{-1}$ is an initial subpath of $y_{+}$. Then $\ell\left(u_{1}^{-1} p_{1}\right)$ is counter-regular, $\ell\left(p_{2} u_{2}^{-1}\right)$ is regular, and $\ell\left(u_{2}^{-1} v^{-1} u_{1}^{-1}\right)$ is regular or counter-regular. If $\ell\left(u_{2}^{-1} v^{-1} u_{1}^{-1}\right)$ is regular, then $\ell\left(p_{2} u_{2}^{-1} v^{-1} u_{1}^{-1}\right)$ is regular. If $\ell\left(u_{2}^{-1} v^{-1} u_{1}^{-1}\right)$ is counter-regular, then $\ell\left(u_{2}^{-1} v^{-1} u_{1}^{-1} p_{1}\right)$ is counter-regular. The cycle $\left\langle p_{1} p_{2} u_{2}^{-1} v^{-1} u_{1}^{-1}\right\rangle$ is the contour of a disc subdiagram of $\Delta$ containing the face $\Pi_{2}$ but not containing the face $\Pi_{1}$. This contradicts the minimality of $\Delta$.

Case 10: $u_{1}$ is a subpath of $y_{-}^{-1}$, and $u_{2}$ is a subpath of $s_{1+}^{\prime-1}$. Contradiction is obtained as in Case 9.

Case 11: $u_{1}$ is a subpath of $y_{+}^{-1}$, and $u_{2}$ is a subpath of $s_{1-}^{\prime-1}$. Let $p_{1}$ and $p_{2}$ be the paths such that $u_{2}^{-1} p_{1}$ is a terminal subpath of $s_{1-}^{\prime}$, and $p_{2} u_{1}^{-1}$ is an initial subpath of $y_{+}$. Then $\ell\left(u_{2}^{-1} p_{1}\right)$ is counter-regular, $\ell\left(p_{2} u_{1}^{-1}\right)$ is regular, and $\ell\left(u_{1} v u_{2}\right)$ is regular or counter-regular. If $\ell\left(u_{1} v u_{2}\right)$ is regular, then the reduced form of $\ell\left(p_{2} v\right)$ is regular. If $\ell\left(u_{1} v u_{2}\right)$ is counter-regular, then the reduced form of $\ell\left(v p_{1}\right)$ is counterregular. The cycle $\left\langle p_{1} p_{2} v\right\rangle$ is the contour of a disc subdiagram of $\Delta$ not containing the faces $\Pi_{1}$ and $\Pi_{2}$. By Lemma 5.4, $\left\langle p_{1} p_{2} v\right\rangle$ is the contour of a degenerate disc subdiagram. Since $s_{1-}^{\prime} y_{+}$and $v$ are reduced, $v=\left(p_{1} p_{2}\right)^{-1}$. Therefore, $u_{1} v u_{2}$ is a subpath of $\left(s_{1-}^{\prime} y_{+}\right)^{-1}$. This gives a contradiction. 


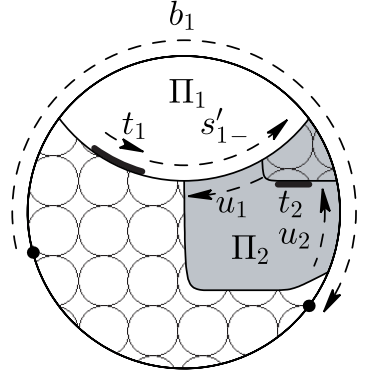

Case 9

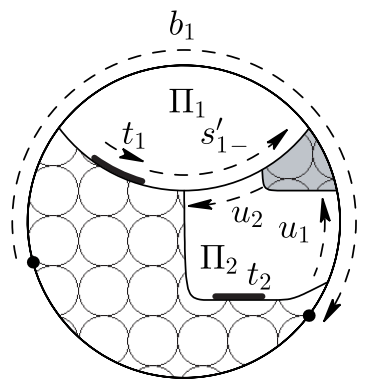

Case 11

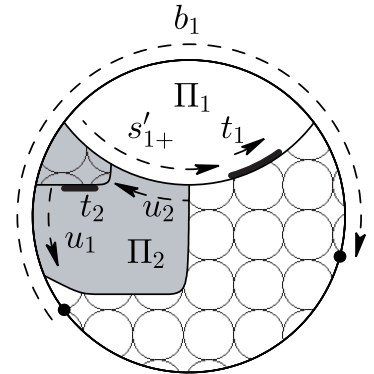

Case 10

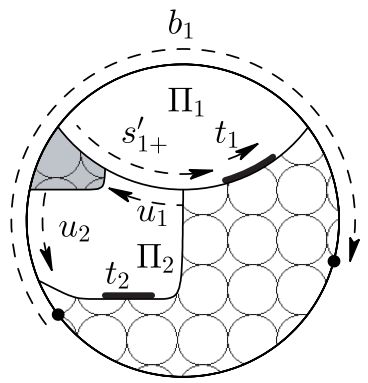

Case 12

Figure 7. Cases 9-12, Lemma 5.10.

Case 12: $u_{1}$ is a subpath of $s_{1+}^{\prime-1}$, and $u_{2}$ is a subpath of $y_{-}^{-1}$. Arguing as in Case 11, one obtains that $u_{1} v u_{2}$ is a subpath of $\left(y_{-} s_{1+}^{\prime}\right)^{-1}$. This gives a contradiction.

Case 13: $u_{1}$ is a subpath of $s_{1-}^{\prime-1}$, and $u_{2}$ is a subpath of $b_{2}^{\prime-1}$. Let $p_{1}$ and $p_{2}$ be the paths such that $p_{1} u_{1}$ is an initial subpath of $y_{-} s_{1}^{-1} s_{1-}^{\prime-1}$, and $u_{2}^{-1} p_{2}$ is a terminal subpath of $b_{2}^{\prime}$. Then $\ell\left(p_{1} u_{1}\right)$ is regular, $\ell\left(u_{2}^{-1} p_{2}\right)$ is counter-regular, and $\ell\left(u_{1} v u_{2}\right)$ is regular or counter-regular. If $\ell\left(u_{1} v u_{2}\right)$ is regular, then $\ell\left(p_{1} u_{1} v\right)$ is regular. If $\ell\left(u_{1} v u_{2}\right)$ is counter-regular, then the reduced form of $\ell\left(u_{1} v p_{2}\right)$ is counter-regular. The cycle $\left\langle p_{2} p_{1} u_{1} v\right\rangle$ is the contour of a disc subdiagram of $\Delta$ containing the face $\Pi_{1}$ but not containing the face $\Pi_{2}$. This contradicts the minimality of $\Delta$.

Case 14: $u_{1}$ is a subpath of $b_{2}^{-1}$, and $u_{2}$ is a subpath of $s_{1+}^{\prime-1}$. Contradiction is obtained as in Case 13.

Case 15: $u_{1}$ is a subpath of $b_{2}^{\prime-1}$, and $u_{2}$ is a subpath of $s_{1-}^{\prime-1}$. Let $p_{1}$ and $p_{2}$ be the paths such that $u_{1}^{-1} p_{1}$ is a terminal subpath of $b_{2}^{\prime}$, and $p_{2} u_{2}$ is an initial subpath of $y_{-} s_{1}^{\prime-1} s_{1-}^{\prime-1}$. The cyclic path $p_{1} p_{2} v^{-1} u_{1}^{-1}$ is cyclically reduced. Suppose it is not simple. Then at least one of the following two subcases takes place:

(a) there is a subpath of $v$ or $p_{1}^{-1}$ which represents the contour of a proper simple disc subdiagram of $\Delta$, or

(b) there are paths $v^{\prime}$ and $p_{2}^{\prime}$ such that $\left\langle v^{\prime} p_{2}^{\prime-1}\right\rangle$ is the contour of a proper simple disc subdiagram of $\Delta$, and $v^{\prime}$ and $p_{2}^{\prime}$ are terminal subpaths of $v$ and $p_{2}$, respectively.

Lemma 5.4 yields a contradiction in both subcases. Hence, $p_{1} p_{2} v^{-1} u_{1}^{-1}$ is a simple path. Therefore, it represents the contour of a disc subdiagram of $\Delta$ containing the 


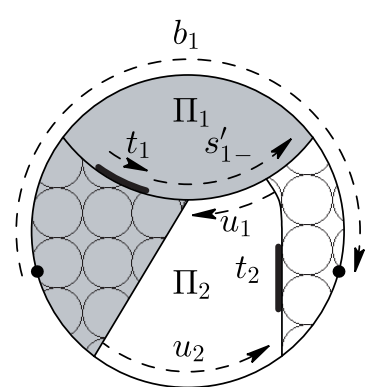

Case 13

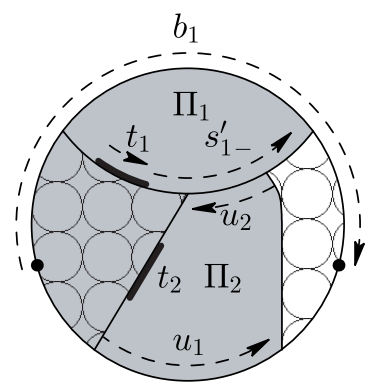

Case 15

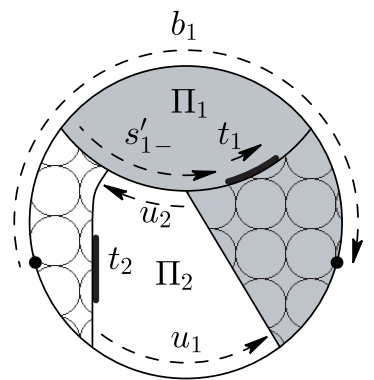

Case 14

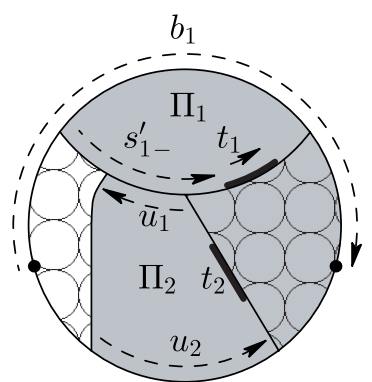

Case 16

Figure 8. Cases 13-16, Lemma 5.10.

faces $\Pi_{1}$ and $\Pi_{2}$. The label of $u_{1}^{-1} p_{1}$ is counter-regular, the label of $p_{2} u_{2}$ is regular, and the label of $u_{2}^{-1} v^{-1} u_{1}^{-1}$ is regular or counter-regular. If $\ell\left(u_{2}^{-1} v^{-1} u_{1}^{-1}\right)$ is regular, then the reduced form of $\ell\left(p_{2} v^{-1} u_{1}^{-1}\right)$ is regular. If $\ell\left(u_{2}^{-1} v^{-1} u_{1}^{-1}\right)$ is counterregular, then $\ell\left(v^{-1} u_{1}^{-1} p_{1}\right)$ is counter-regular. By Lemma 5.4, $\left\langle p_{1} p_{2} v^{-1} u_{1}^{-1}\right\rangle=\partial \Delta$. Therefore, $u_{1} v u_{2}$ is a subpath of $\left(s_{1-}^{\prime} q_{1}\right)^{-1}$. This gives a contradiction.

Case 16: $u_{1}$ is a subpath of $s_{1+}^{\prime-1}$, and $u_{2}$ is a subpath of $b_{2}^{\prime-1}$. Arguing as in Case 15 , one obtains that $u_{1} v u_{2}$ is a subpath of $\left(q_{1} s_{1+}^{\prime}\right)^{-1}$. This gives a contradiction.

A contradiction is obtained in each of the considered cases, and no other case is possible. Thus, $s_{2}$ and $\left(s_{1-}^{\prime} q_{1} s_{1+}^{\prime}\right)^{-1}$ have exactly one maximal common nontrivial subpath.

Lemma 5.11. If $\Pi$ is a face of $\Delta$, the rank of every other face of $\Delta$ is less than the rank of $\Pi, s$ is the maximal selected subpath of $\partial \Pi$, and $e_{1}$ and $e_{n}$ are, respectively, the initial and the terminal subpaths of $s$ of length $|s| / n$, then all oriented edges of $e_{1}$ or all oriented edges of $e_{n}$ are internal in $\Delta$.

Proof. Observe that proving that all oriented edges of $e_{1}$ or all oriented edges of $e_{n}$ are internal in $\Delta$ is equivalent to proving that all oriented edges of $e_{n}^{-1}$ or all oriented edges of $e_{1}^{-1}$ are internal in the mirror copy of $\Delta$.

Suppose $\Pi, s, e_{1}, e_{n}$ are such as in the hypotheses of the lemma. Assume that $\ell(s)$ is regular (if it is not, pass from $\Delta, s, e_{1}, e_{n}$ to the mirror copy of $\Delta, s^{-1}, e_{n}^{-1}$, $e_{1}^{-1}$, respectively). 

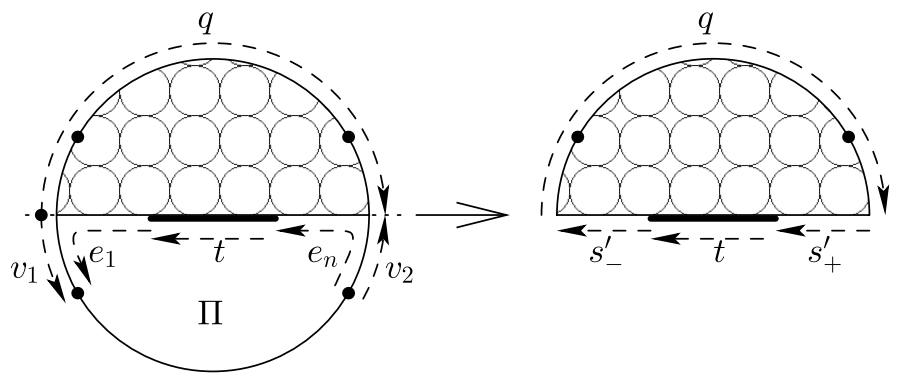

Figure 9. The face $\Pi$ in $\Delta$ and the subdiagram $\Delta^{\prime}$, Lemma 5.11 .

Suppose that some oriented edge of $e_{1}$ and some oriented edge of $e_{n}$ are external in $\Delta$ (see Figure 9).

Let $s^{\prime}$ be the maximal selected external oriented arc of $\Delta$ that is a subpath of $s$. Let $s_{-}^{\prime}$ and $s_{+}^{\prime}$ be such paths that $s=s_{-}^{\prime} s^{\prime} s_{+}^{\prime}$. Then $s_{-}^{\prime}$ is a proper initial subpath of $e_{1}$, and $s_{+}^{\prime}$ is a proper terminal subpath of $e_{n}$. Let $v_{1}$ and $v_{2}$ be the initial and the terminal subpaths of $s^{\prime}$ such that $s_{-}^{\prime} v_{1}=e_{1}$ and $v_{2} s_{+}^{\prime}=e_{n}$. Let $t$ be the path such that $\langle s t\rangle=\partial \Pi$. Let $q$ be the path such that $\left\langle q s^{\prime-1}\right\rangle=\partial \Delta$. Let $\Delta^{\prime}$ be the disc subdiagram of $\Delta$ obtained by removing the face $\Pi$ and all edges that lie on $s^{\prime}$ together with all intermediate vertices of $s^{\prime}$. The contour of $\Delta^{\prime}$ is $\left\langle s_{+}^{\prime} t s_{-}^{\prime} q\right\rangle$.

Let $j=\operatorname{rank}(\Pi)$. Then $\ell\left(e_{1}\right)=x_{1}^{m_{j}}$ and $\ell\left(e_{n}\right)=x_{n}^{m_{j}}$. The label of the first oriented edge of $s^{\prime}$ is $x_{1}$; the label of the last oriented edge of $s^{\prime}$ is $x_{n}$. Therefore, $s^{\prime}$ is a subpath of $b_{2}^{-1}$. Note that $v_{1}^{-1}$ is an initial subpath of $l_{1}, v_{2}^{-1}$ is a terminal subpath of $l_{2}$, and $v_{1}^{-1} q v_{2}^{-1}=b_{1}$. The reduced form of $\ell\left(s_{-}^{\prime} q s_{+}^{\prime}\right)$ is regular since $\ell(q)$ is regular, $\ell\left(s_{-}^{\prime}\right)$ is a power of $x_{1}$, and $\ell\left(s_{+}^{\prime}\right)$ is a power of $x_{n}$. As it has been assumed, $\operatorname{rank}\left(\Pi^{\prime}\right)<j$ for every face $\Pi^{\prime}$ of $\Delta^{\prime}$. Hence, the group word $w_{j}=\ell\left(t^{-1}\right)$ equals a regular word (the reduced form of $\ell\left(s_{-}^{\prime} q s_{+}^{\prime}\right)$ ) modulo the relations $r=1$, $r \in \mathcal{R}_{j-1}$. This contradicts the choice of $w_{i}$.

Lemma 5.12. There are two distinct faces $\Pi_{1}$ and $\Pi_{2}$, and paths $s_{1}, s_{1}^{\prime}, s_{1-}^{\prime}, s_{1+}^{\prime}$, $s_{2}, s_{2}^{\prime}, s_{2-}^{\prime}, s_{2+}^{\prime}, z_{1}, z_{2}$ in $\Delta$ such that:

- $s_{1}$ and $s_{2}$ are the maximal selected subpaths of $\partial \Pi_{1}$ and $\partial \Pi_{2}$, respectively;

- $\ell\left(s_{1}\right)$ is counter-regular, $\ell\left(s_{2}\right)$ is regular;

- $s_{i}=s_{i-}^{\prime} s_{i}^{\prime} s_{i+}^{\prime}$ for both $i=1$ and $i=2$;

- both $s_{1}^{\prime}$ and $s_{2}^{\prime}$ are maximal selected external oriented arcs of $\Delta$;

- $\left\langle z_{1} s_{1}^{\prime-1} z_{2} s_{2}^{\prime-1}\right\rangle=\partial \Delta$

- all basic letters of $\ell\left(s_{2-}^{\prime} z_{1} s_{1+}^{\prime}\right)$ are in $\left\{x_{1}, \ldots, x_{21}\right\}$, and all basic letters of $\ell\left(s_{1-}^{\prime} z_{2} s_{2+}^{\prime}\right)$ are in $\left\{x_{n-20}, \ldots, x_{n}\right\}$.

Proof. Observe that this lemma is equivalent to the analogous lemma about the mirror copy of $\Delta$, i.e., to the statement obtained from this lemma by substituting "the mirror copy of $\Delta$ " for " $\Delta$. ."

Let $\Pi_{1}$ be a face of $\Delta$ such as in Lemma 5.9. Let $s_{1}$ be the maximal selected subpath of $\partial \Pi_{1}$. If $\ell\left(s_{1}\right)$ is regular, pass from $\Delta$ and $s_{1}$ to the mirror copy of $\Delta$ and $s_{1}^{-1}$, respectively. Hence, $\ell\left(s_{1}\right)$ shall be assumed to be counter-regular. Let $s_{1}^{\prime}$ be the maximal selected external oriented arc of $\Delta$ that is a subpath of $s_{1}$. Let $s_{1-}^{\prime}$ and $s_{1+}^{\prime}$ be the paths such that $s_{1}=s_{1-}^{\prime} s_{1}^{\prime} s_{1+}^{\prime}$. Since all of the basic letters 
$x_{21}, x_{22}, \ldots, x_{n-20}$ occur in $\ell\left(s_{1}^{\prime}\right)$, it follows that all basic letters of $\ell\left(s_{1-}^{\prime}\right)$ are in $\left\{x_{n-20}, \ldots, x_{n}\right\}$, and all basic letters of $\ell\left(s_{1+}^{\prime}\right)$ are in $\left\{x_{1}, \ldots, x_{21}\right\}$.

Let $t_{1}$ be the path such that $\left\langle s_{1} t_{1}\right\rangle=\partial \Pi_{1}$. Let $q_{1}$ be the path such that $\left\langle s_{1}^{\prime-1} q_{1}\right\rangle=\partial \Delta$. Let $\Delta^{\prime}$ be the disc S-subdiagram of $\Delta$ obtained by removing the face $\Pi_{1}$ and all edges that lie on $s_{1}^{\prime}$ together with all intermediate vertices of $s_{1}^{\prime}$. The contour of $\Delta^{\prime}$ is $\left\langle s_{1+}^{\prime} t_{1} s_{1-}^{\prime} q_{1}\right\rangle$.

It shall be shown that there is a face $\Pi$ of $\Delta^{\prime}$ such that $\Pi$ is incident to no fewer than $(1-21 / n)|\partial \Pi|$ selected external edges of $\Delta^{\prime}$ that do not lie on $t_{1}$. Consider 2 cases:

Case 1: there is a face $\Pi$ in $\Delta^{\prime}$ such that $\operatorname{rank}(\Pi) \geq \operatorname{rank}\left(\Pi_{1}\right)$. Then let $\Phi$ be the maximal simple disc S-subdiagram of $\Delta^{\prime}$ that contains such a face $\Pi$. Let $\Sigma$ be the sum of the degrees of all the faces of $\Phi$. Then $\Sigma \geq|\partial \Pi| \geq\left|\partial \Pi_{1}\right|$. By Lemma 5.3, the number of selected external edges of $\Phi$ is at least $(1-2 \mu) \Sigma$. Therefore, the number of selected external edges of $\Phi$ that do not lie on $t_{1}$ is at least

$$
(1-2 \mu) \Sigma-\lambda_{1}\left|\partial \Pi_{1}\right| \geq\left(1-2 \mu-\lambda_{1}\right) \Sigma>\frac{n-21}{n} \Sigma .
$$

Case 2: the rank of every face of $\Delta^{\prime}$ is less than the rank of $\Pi_{1}$. Let $e_{11}$ and $e_{1 n}$ be respectively the initial and terminal subpaths of $s_{1}$ of length $\left|s_{1}\right| / n$. Then

$\ell\left(e_{11}\right)=x_{n}^{-m_{j_{1}}}$, and $\ell\left(e_{1 n}\right)=x_{1}^{-m_{j_{1}}}$. Since the rank of $\Pi_{1}$ is greater than the rank of every other face of $\Delta$, all oriented edges of $e_{11}$ or all oriented edges of $e_{1 n}$ are internal in $\Delta$ by Lemma 5.11. Let $e$ denote $e_{11}$ in the first case or $e_{1 n}$ in the second.

If an edge lies on $e$ and is not incident to any face of $\Delta^{\prime}$, then it also lies on $t_{1}$. Indeed, both (mutually inverse) oriented edges corresponding to such an edge must occur in $\partial \Pi_{1}$; one of them is an oriented edge of $e$, and consequently the other cannot be an oriented edge of $s_{1}$ (two mutually inverse group letters cannot both occur in $\left.\ell\left(s_{1}\right)\right)$ and has to be an oriented edge of $t_{1}$. Since

$$
\left|t_{1}\right|=\left|w_{j_{1}}\right|<m_{j_{1}}=|e|
$$

(see (5.5)), the ratio of the number of edges that lie on $t_{1}$ and are incident with faces of $\Delta^{\prime}$ to the number of edges that lie on $e$ but are also incident with faces of $\Delta^{\prime}$ is not greater than

$$
\frac{\left|t_{1}\right|}{|e|}=\frac{\left|w_{j_{1}}\right|}{m_{j_{1}}} \leq \frac{n \lambda_{1}}{1-\lambda_{1}}
$$

(see (5.4)). Let $\Phi$ be a maximal simple disc S-subdiagram of $\Delta^{\prime}$ such that the ratio of the number of its (external) edges that lie on $t_{1}$ to the number of its (external) edges that lie on $e$ is not greater than

$$
\frac{n \lambda_{1}}{1-\lambda_{1}}
$$

Let $\Sigma$ be the sum of the degrees of all the faces of $\Phi$. Since all edges that lie on $e$ are labelled with a same basic letter, the number of edges of $\Phi$ that lie on $e$ is less than

$$
\left(\lambda_{1}+\frac{1-\lambda_{1}}{n}\right) \Sigma
$$

(because the number of edges with a same label incident to a given face $\Pi$ of $\Delta$ is less than $\left.\left(\lambda_{1}+\left(1-\lambda_{1}\right) / n\right)|\partial \Pi|\right)$. By Lemma 5.3, the number of selected external edges of $\Phi$ is at least $(1-2 \mu) \Sigma$. Therefore, the number of selected external edges 
of $\Phi$ that do not lie on $t_{1}$ is greater than

$$
\left(1-2 \mu-\frac{n \lambda_{1}}{1-\lambda_{1}}\left(\lambda_{1}+\frac{1-\lambda_{1}}{n}\right)\right) \Sigma=\left(1-2 \mu-\lambda_{1}-\frac{n \lambda_{1}^{2}}{1-\lambda_{1}}\right) \Sigma>\frac{n-21}{n} \Sigma
$$

$(\operatorname{see}(5.2))$.

In both cases, there is a face $\Pi$ in $\Phi$ such that $\Pi$ is incident to at least $(1-21 / n)|\partial \Pi|$ selected external edges of $\Phi$ (of $\Delta^{\prime}$ ) that do not lie on $t_{1}$. Let $\Pi_{2}$ be such a face. Let $s_{2}$ be the maximal selected subpath of $\partial \Pi_{2}$. Then the paths $s_{2}$ and $\left(s_{1-}^{\prime} q_{1} s_{1+}^{\prime}\right)^{-1}$ have at least $(1-21 / n)\left|\partial \Pi_{2}\right|$ common oriented edges. Let $\tilde{s}_{2}$ be the maximal common nontrivial subpath of $s_{2}$ and $\left(s_{1-}^{\prime} q_{1} s_{1+}^{\prime}\right)^{-1}$ (see Lemma 5.10). Note that $\tilde{s}_{2}$ is a selected external oriented arc of $\Delta^{\prime}$. Every selected external edge of $\Delta^{\prime}$ incident to $\Pi_{2}$ lies either on $t_{1}$ or on $\tilde{s}_{2}$. Therefore,

$$
\left|\tilde{s}_{2}\right| \geq \frac{n-21}{n}\left|\partial \Pi_{2}\right|>\frac{n-21}{n}\left|s_{2}\right|
$$

Therefore, $\ell\left(\tilde{s}_{2}\right)$ has at least $n-20$ distinct basic letters, which implies that each of the basic letters $x_{21}, \ldots, x_{n-20}$ occurs in it. The basic letters $x_{22}, \ldots, x_{n-21}$ do not occur on $s_{1-}^{\prime}$ nor on $s_{1+}^{\prime}$, but they occur in $\ell\left(\tilde{s}_{2}\right)$. Hence, the paths $\tilde{s}_{2}$ and $q_{1}^{-1}$ have common oriented edges. Therefore, $\Pi_{2}$ is incident to some selected external edges of $\Delta$.

Let $s_{2}^{\prime}$ be the maximal selected external oriented arc of $\Delta$ that is a subpath of $s_{2}$. Then $s_{2}^{\prime}$ is the maximal common subpath of $\tilde{s}_{2}$ and $q_{1}^{-1}$. All of the basic letters $x_{22}, \ldots, x_{n-21}$ occur in $\ell\left(s_{2}^{\prime}\right)$ (since they occur in $\ell\left(\tilde{s}_{2}\right)$ but neither in $\ell\left(s_{1-}^{\prime}\right)$ nor in $\left.\ell\left(s_{1+}^{\prime}\right)\right)$.

Since $\ell\left(s_{1}\right)$ is counter-regular, $s_{1}^{\prime}$ is a subpath of $b_{1}^{-1}$. Therefore, $s_{2}^{\prime}$ is a subpath of $b_{2}^{-1}$, and $\ell\left(s_{2}\right)$ is regular.

Let $s_{2-}^{\prime}$ and $s_{2+}^{\prime}$ be the paths such that $s_{2}=s_{2-}^{\prime} s_{2}^{\prime} s_{2+}^{\prime}$. Let $z_{1}$ and $z_{2}$ be the paths such that $\left\langle z_{1} s_{1}^{\prime-1} z_{2} s_{2}^{\prime-1}\right\rangle=\partial \Delta$. It shall be proved that all basic letters of $\ell\left(s_{2-}^{\prime}\right)$ and $\ell\left(z_{1}\right)$ are among $x_{1}, \ldots, x_{21}$, and all basic letters of $\ell\left(s_{2+}^{\prime}\right)$ and $\ell\left(z_{2}\right)$ are among $x_{n-20}, \ldots, x_{n}$.

First, consider $s_{2-}^{\prime}$ and $z_{1}$.

Case 1: $\tilde{s}_{2}$ has no common oriented edges with $s_{1+}^{\prime-1}$. Then $s_{2}^{\prime}$ is an initial subpath of $\tilde{s}_{2}$. Therefore, the basic letter $x_{21}$, along with $x_{22}, \ldots, x_{n-21}$, occurs in $\ell\left(s_{2}^{\prime}\right)$. Hence, all basic letters of $\ell\left(s_{2-}^{\prime}\right)$ are in $\left\{x_{1}, \ldots, x_{21}\right\}$. The label of every edge that lies on both $z_{1}$ and $b_{1}$ is in $\left\{x_{1}, \ldots, x_{21}\right\}$ since the basic letter $x_{21}$ occurs in $\ell\left(s_{1}^{\prime}\right)$, and the only maximal common subpath of $z_{1} s_{1}^{\prime-1}$ and $b_{1}$ is an initial subpath of $b_{1}$. The label of every edge that lies on both $z_{1}$ and $b_{2}$ is in $\left\{x_{1}, \ldots, x_{21}\right\}$ since the basic letter $x_{21}$ occurs in $\ell\left(s_{2}^{\prime}\right)$, and the only maximal common subpath of $s_{2}^{\prime-1} z_{1}$ and $b_{2}$ is a terminal subpath of $b_{2}$.

Case 2: $\tilde{s}_{2}$ has at least one oriented edge in common with $s_{1+}^{\prime-1}$. Then some nontrivial terminal subpath of $s_{1+}^{\prime-1}$ is an initial subpath of $\tilde{s}_{2}$, and $z_{1}$ is trivial. The terminal subpath of $s_{1+}^{\prime-1}$ that is an initial subpath of $\tilde{s}_{2}$ is also a terminal subpath of $s_{2-}^{\prime}$. Since $s_{1+}^{\prime-1}$ and $s_{2-}^{\prime}$ have a common nontrivial terminal subpath, the sets of basic letters of their labels coincide. Therefore, all basic letters of $\ell\left(s_{2-}^{\prime}\right)$ are in $\left\{x_{1}, \ldots, x_{21}\right\}$. The label of $z_{1}$ is empty.

Second, consider $s_{2+}^{\prime}$ and $z_{2}$ in the same manner.

Case 1: $\tilde{s}_{2}$ has no common oriented edges with $s_{1-}^{\prime-1}$. Then $s_{2}^{\prime}$ is a terminal subpath of $\tilde{s}_{2}$. Therefore, the basic letter $x_{n-20}$, along with $x_{22}, \ldots, x_{n-21}$, occurs in $\ell\left(s_{2}^{\prime}\right)$. Hence, all basic letters of $\ell\left(s_{2+}^{\prime}\right)$ are in $\left\{x_{n-20}, \ldots, x_{n}\right\}$. The label of 
every edge that lies on both $z_{2}$ and $b_{1}$ is in $\left\{x_{n-20}, \ldots, x_{n}\right\}$ since the basic letter $x_{n-20}$ occurs in $\ell\left(s_{1}^{\prime}\right)$, and the only maximal common subpath of $s_{1}^{\prime-1} z_{2}$ and $b_{1}$ is a terminal subpath of $b_{1}$. The label of every edge that lies on both $z_{2}$ and $b_{2}$ is in $\left\{x_{n-20}, \ldots, x_{n}\right\}$ since the basic letter $x_{n-20}$ occurs in $\ell\left(s_{2}^{\prime}\right)$, and the only maximal common subpath of $z_{2} s_{2}^{\prime-1}$ and $b_{2}$ is an initial subpath of $b_{2}$.

Case 2: $\quad \tilde{s}_{2}$ has at least one oriented edge in common with $s_{1-}^{\prime-1}$. Then some nontrivial initial subpath of $s_{1-}^{\prime-1}$ is a terminal subpath of $\tilde{s}_{2}$, and $z_{2}$ is trivial. The initial subpath of $s_{1-}^{-1}$ that is a terminal subpath of $\tilde{s}_{2}$ is also an initial subpath of $s_{2+}^{\prime}$. Since $s_{1-}^{\prime-1}$ and $s_{2+}^{\prime}$ have a common nontrivial initial subpath, the sets of basic letters of their labels coincide. Therefore, all basic letters of $\ell\left(s_{2+}^{\prime}\right)$ are in $\left\{x_{n-20}, \ldots, x_{n}\right\}$. The label of $z_{2}$ is empty.

Clearly, the faces $\Pi_{1}, \Pi_{2}$ and the paths $s_{1}, s_{1}^{\prime}, s_{1-}^{\prime}, s_{1+}^{\prime}, s_{2}, s_{2}^{\prime}, s_{2-}^{\prime}, s_{2+}^{\prime}, z_{1}, z_{2}$ are desired ones.

Lemma 5.13. The diagram $\Delta$ has more than 2 faces.

Proof. Note that $\Delta$ obviously has the same number of faces as its mirror copy.

Suppose the statement is not true, i.e., $\Delta$ has no more than 2 faces. (Then, by Lemma 5.5, it has exactly 2 faces.)

Let $\Pi_{1}$ and $\Pi_{2}$ be such faces of $\Delta$ as in Lemma 5.12. Then the diagram $\Delta$ consists of the faces $\Pi_{1}$ and $\Pi_{2}$ attached to each other along a common arc. If $\operatorname{rank}\left(\Pi_{2}\right)>\operatorname{rank}\left(\Pi_{1}\right)$, pass to the mirror copy of $\Delta$ and interchange the roles of $\Pi_{1}$ and $\Pi_{2}$. Hence, it shall be assumed that $\operatorname{rank}\left(\Pi_{1}\right) \geq \operatorname{rank}\left(\Pi_{2}\right)$.

Let $s_{1}, s_{1}^{\prime}, s_{1-}^{\prime}, s_{1+}^{\prime}, s_{2}, s_{2}^{\prime}, s_{2-}^{\prime}, s_{2+}^{\prime}$ be the subpaths of $\partial \Pi_{1}$ and $\partial \Pi_{2}$ such as in Lemma 5.12. Then all basic letters of the labels of $s_{1-}^{\prime}, s_{1+}^{\prime}, s_{2-}^{\prime}, s_{2+}^{\prime}$ are in $\left\{x_{1}, \ldots, x_{21}\right\} \cup\left\{x_{n-20}, \ldots, x_{n}\right\}$, and $s_{i}^{\prime}$ is a subpath of $b_{i}^{-1}$ for $i=1$ and $i=2$.

There are exactly two (mutually inverse) maximal internal oriented arcs in $\Delta$. Denote the one that is a subpath of $\partial \Pi_{1}$ by $u$.

Let $t_{1}$ and $t_{2}$ be the path such that $\left\langle s_{1} t_{1}\right\rangle=\partial \Pi_{1}$ and $\left\langle s_{2} t_{2}\right\rangle=\partial \Pi_{2}$. Each of the faces $\Pi_{1}$ and $\Pi_{2}$ is incident to exactly one maximal selected external arc of $\Delta$. Therefore, each of the paths $t_{1}$ and $t_{2}$ has a common vertex with $u$.

Suppose $t_{1}$ and $t_{2}$ do not have common vertices. Then either some (proper) nontrivial initial subpath of $s_{1}$ is inverse to a (proper) initial subpath of $s_{2}$, or some (proper) nontrivial terminal subpath of $s_{1}$ is inverse to a (proper) terminal subpath of $s_{2}$. Both cases are impossible since no proper nontrivial prefix of $\ell\left(s_{2}\right)$ can be a suffix of $\ell\left(s_{1}^{-1}\right)$, and no proper nontrivial suffix of $\ell\left(s_{2}\right)$ can be a prefix of $\ell\left(s_{1}^{-1}\right)$. (A contradiction may also be obtained by applying Lemma 5.10.) Hence, the paths $t_{1}$ and $t_{2}$ have at least one common vertex.

Let $j_{1}=\operatorname{rank}\left(\Pi_{1}\right)$ and $j_{2}=\operatorname{rank}\left(\Pi_{2}\right)$. Let $e_{i 1}$ and $e_{i n}$ be respectively the initial and the terminal subpaths of $s_{i}$ of length $m_{j_{i}}=\left|s_{i}\right| / n$ for $i=1$ and $i=2$. Then $\ell\left(e_{11}\right)=x_{n}^{-m_{j_{1}}}, \ell\left(e_{1 n}\right)=x_{1}^{-m_{j_{1}}}, \ell\left(e_{21}\right)=x_{1}^{m_{j_{2}}}$, and $\ell\left(e_{2 n}\right)=x_{n}^{m_{j_{2}}}$.

At most one of the two group letters $x_{1}^{ \pm 1}$ and at most one of $x_{n}^{ \pm 1}$ can occur as labels of oriented edges of $\partial \Delta$. Therefore, at least one of the paths $e_{1 n}$ and $e_{21}$ and at least one of the paths $e_{11}$ and $e_{2 n}$ do not have external oriented edges in $\Delta$.

Suppose $\operatorname{rank}\left(\Pi_{1}\right)=\operatorname{rank}\left(\Pi_{2}\right)$. Then

$$
\left|e_{11}\right|=\left|e_{1 n}\right|=\left|e_{21}\right|=\left|e_{2 n}\right|=m_{j_{1}} \geq\left|w_{j_{1}}\right|=\left|t_{1}\right|=\left|t_{2}\right| .
$$

Recall that $w_{j_{1}}$ does not start with $x_{1}^{ \pm 1}$, does not end with $x_{n}^{ \pm 1}$, and is not a letter power. Therefore, every subpath of either $\partial \Pi_{1}$ or $\partial \Pi_{2}$ labelled with a letter power 


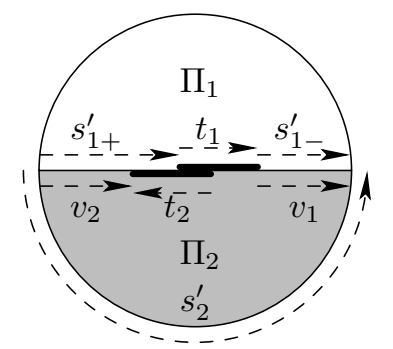

Case 1

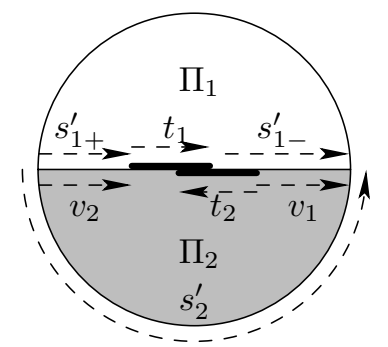

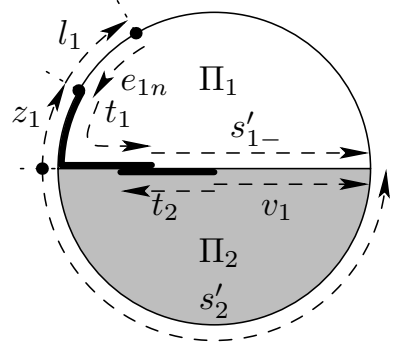

Case 2

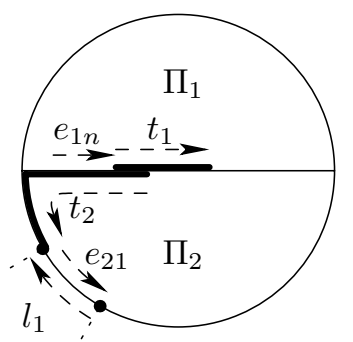

Case 4

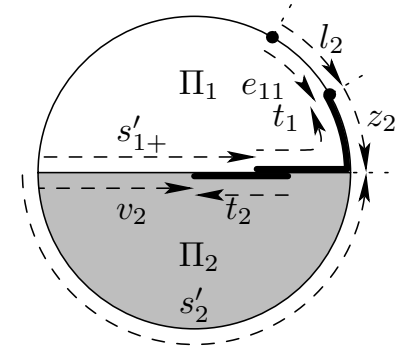

Case 3

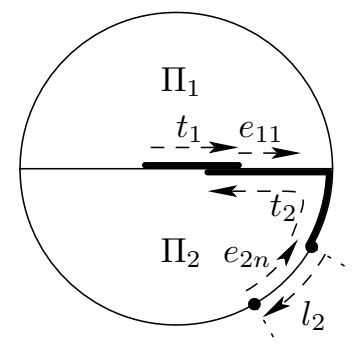

Case 5

Figure 10. Cases 1-5, Lemma 5.13.

has length of at most $m_{j_{1}}$. Moreover, for every $i$, there exist exactly one subpath of $\partial \Pi_{1}$ and exactly one subpath of $\partial \Pi_{2}$ of length $m_{j_{1}}$ labelled with powers of $x_{i}$ (i.e., with $\left.x_{i}^{ \pm m_{j_{1}}}\right)$. If $e_{1 n}$ has no external oriented edges in $\Delta$, then it is a subpath of $u$. If $e_{21}$ has no external oriented edges in $\Delta$, then it is a subpath of $u^{-1}$. In either case $u$ has a subpath labelled with $x_{1}^{-m_{j_{1}}}$, and the pair $\left\{\Pi_{1}, \Pi_{2}\right\}$ is immediately cancellable. This contradicts Lemma 5.1. Hence, $\operatorname{rank}\left(\Pi_{1}\right)>\operatorname{rank}\left(\Pi_{2}\right)$.

Let $\Delta^{\prime}$ be the disc S-subdiagram of $\Delta$ obtained by removing the face $\Pi_{1}$ and all edges and intermediate vertices of $s_{1}^{\prime}$. The only face of $\Delta^{\prime}$ is $\Pi_{2}$.

By Lemma 5.11, all oriented edges of $e_{11}$ or all oriented edges of $e_{1 n}$ are internal in $\Delta$. Consider the following 5 cases (see Figure 10):

(Notice that Case 3 is "symmetric" with Case 2, and Case 5 -with Case 4.)

Case 1: each of the paths $e_{11}, e_{1 n}, e_{21}$, and $e_{2 n}$ has an internal oriented edge. Then the paths $s_{1-}^{\prime}$ and $s_{2+}^{\prime-1}$ have a common nontrivial terminal subpath, the paths 
$s_{1+}^{\prime}$ and $s_{2-}^{\prime-1}$ have a common nontrivial initial subpath, and $\partial \Delta=\left\langle s_{1}^{\prime-1} s_{2}^{\prime-1}\right\rangle$. Let $v_{1}$ be a common nontrivial terminal subpath of $s_{1-}^{\prime}$ and $s_{2+}^{\prime-1}$, and let $v_{2}$ be a common nontrivial initial subpath of $s_{1+}^{\prime}$ and $s_{2-}^{\prime-1}$. The contour of $\Delta^{\prime}$ is $\left\langle s_{1+}^{\prime} t_{1} s_{1-}^{\prime} s_{2}^{\prime-1}\right\rangle$. The label of $s_{1-}^{\prime} s_{2}^{\prime-1} s_{1+}^{\prime}$ is counter-regular because the labels of $s_{1-}^{\prime}, v_{1} s_{2}^{\prime-1} v_{2}$, and $s_{1+}^{\prime}$ are such. Hence, the group word $w_{j_{1}}=\ell\left(t_{1}\right)$ equals the regular word $\ell\left(s_{1-}^{\prime} s_{2}^{\prime-1} s_{1+}^{\prime}\right)^{-1}$ modulo the relation $r_{j_{2}}=1$. This contradicts the choice of $w_{j_{1}}$.

Case 2: all oriented edges of $e_{1 n}$ are external (consequently, all oriented edges of $e_{11}$ and $e_{21}$ are internal), and some oriented edge of $e_{2 n}$ is internal. Then the paths $s_{1-}^{\prime}$ and $s_{2+}^{\prime-1}$ have a common nontrivial terminal subpath. Let $v_{1}$ be a common nontrivial terminal subpath of $s_{1-}^{\prime}$ and $s_{2+}^{\prime-1}$. Let $z_{1}$ be the path such that $\left\langle z_{1} s_{1}^{\prime-1} s_{2}^{\prime-1}\right\rangle=\partial \Delta$. Then $z_{1}^{-1}$ is an initial subpath of $t_{1}$. Since $\ell\left(e_{1 n}\right)$ is a power of $x_{1}$, the path $e_{1 n}^{-1}$ is a (terminal) subpath of $b_{2}$. Therefore, $s_{2}^{\prime-1} z_{1} e_{1 n}^{-1}$ is a subpath of $b_{2}$. The contour of $\Delta^{\prime}$ is $\left\langle z_{1} t_{1} s_{1-}^{\prime} s_{2}^{\prime-1}\right\rangle$. The label of $s_{1-}^{\prime} s_{2}^{\prime-1} z_{1}$ is counter-regular because the labels of $s_{1-}^{\prime}, v_{1} s_{2}^{\prime-1}$, and $s_{2}^{\prime-1} z_{1}$ are such. Hence, the group word $w_{j_{1}}=\ell\left(t_{1}\right)$ equals the regular word $\ell\left(s_{1-}^{\prime} s_{2}^{\prime-1} z_{1}\right)^{-1}$ modulo the relation $r_{j_{2}}=1$. This contradicts the choice of $w_{j_{1}}$.

Case 3: all oriented edges of $e_{11}$ are external (consequently, all oriented edges of $e_{1 n}$ and $e_{2 n}$ are internal), and some oriented edge of $e_{21}$ is internal. Then the paths $s_{1+}^{\prime}$ and $s_{2-}^{\prime-1}$ have a common nontrivial initial subpath. Let $v_{2}$ be a common nontrivial initial subpath of $s_{1+}^{\prime}$ and $s_{2-}^{\prime-1}$. Let $z_{2}$ be the path such that $\left\langle s_{1}^{\prime-1} z_{2} s_{2}^{\prime-1}\right\rangle=\partial \Delta$. Then $z_{2}^{-1}$ is a terminal subpath of $t_{1}$. Since $\ell\left(e_{11}\right)$ is a power of $x_{n}$, the path $e_{11}^{-1}$ is an (initial) subpath of $b_{2}$. Therefore, $e_{11}^{-1} z_{2} s_{2}^{-1}$ is a subpath of $b_{2}$. The contour of $\Delta^{\prime}$ is $\left\langle s_{1+}^{\prime} t_{1} z_{2} s_{2}^{\prime-1}\right\rangle$. The label of $z_{2} s_{2}^{\prime-1} s_{1+}^{\prime}$ is counter-regular because the labels of $z_{2} s_{2}^{\prime-1}, s_{2}^{\prime-1} v_{2}$, and $s_{1+}^{\prime}$ are such. Hence, the group word $w_{j_{1}}=\ell\left(t_{1}\right)$ equals the regular word $\ell\left(z_{2} s_{2}^{\prime-1} s_{1+}^{\prime}\right)^{-1}$ modulo the relation $r_{j_{2}}=1$. This contradicts the choice of $w_{j_{1}}$.

Case 4: all oriented edges of $e_{21}$ are external. Consequently, all oriented edges of $e_{1 n}$ are internal. Then $e_{1 n}$ is a terminal subpath of $s_{1+}^{\prime}$. The path $s_{1+}^{\prime-1}$ is a subpath of $t_{2}$ (because $t_{2}$ has common vertices with both $e_{21}$ and $t_{1}$ ). Therefore, $\left|t_{2}\right| \geq\left|s_{1+}^{\prime}\right| \geq\left|e_{1 n}\right|=m_{j_{1}}>\left|w_{j_{2}}\right|=\left|t_{2}\right|$ (see (5.5)). This gives a contradiction.

Case 5: all oriented edges of $e_{2 n}$ are external. Consequently, all oriented edges of $e_{11}$ are internal. Then $e_{11}$ is an initial subpath of $s_{1-}^{\prime}$. The path $s_{1-}^{\prime-1}$ is a subpath of $t_{2}$ (because $t_{2}$ has common vertices with both $e_{2 n}$ and $t_{1}$ ). Therefore, $\left|t_{2}\right| \geq\left|s_{1-}^{\prime}\right| \geq\left|e_{11}\right|=m_{j_{1}}>\left|w_{j_{2}}\right|=\left|t_{2}\right|$ (see (5.5)). This gives a contradiction.

No other case is possible. Thus, $\Delta$ has more than 2 faces.

Lemma 5.14. Let $\Pi_{1}$ and $\Pi_{2}$ be distinct faces of $\Delta$ such as in Lemma 5.12. Then the ranks of $\Pi_{1}$ and $\Pi_{2}$ are distinct.

Proof. Let $\Pi_{1}, \Pi_{2}, s_{1}, s_{1}^{\prime}, s_{1-}^{\prime}, s_{1+}^{\prime}, s_{2}, s_{2}^{\prime}, s_{2-}^{\prime}, s_{2+}^{\prime}, z_{1}, z_{2}$ be as in Lemma 5.12. Let $c_{1}=s_{2-}^{\prime} z_{1} s_{1+}^{\prime}$ and $c_{2}=s_{1-}^{\prime} z_{2} s_{2+}^{\prime}$. Let $t_{1}$ and $t_{2}$ be the paths such that $\left\langle s_{1} t_{1}\right\rangle=\partial \Pi_{1}$ and $\left\langle s_{2} t_{2}\right\rangle=\partial \Pi_{2}$. Let $\Delta^{\prime}$ be the disc S-subdiagram of $\Delta$ obtained by removing the faces $\Pi_{1}$ and $\Pi_{2}$ and all edges and intermediate vertices of the paths $s_{1}^{\prime}$ and $s_{2}^{\prime}$ (see Figure 11).

The contour of $\Delta^{\prime}$ is $\left\langle c_{1} t_{1} c_{2} t_{2}\right\rangle$. By Lemma 5.13 , the diagram $\Delta^{\prime}$ is nondegenerate. Therefore, by Lemma 5.4, the label of $c_{1} t_{1} c_{2} t_{2}$ is not freely trivial.

Suppose $\operatorname{rank}\left(\Pi_{1}\right)=\operatorname{rank}\left(\Pi_{2}\right)$. Let $j=\operatorname{rank}\left(\Pi_{1}\right)=\operatorname{rank}\left(\Pi_{2}\right)$. Recall that $\ell\left(t_{1}\right)=w_{j}, \ell\left(t_{2}\right)=w_{j}^{-1}$, all basic letters of $\ell\left(c_{1}\right)$ are in $\left\{x_{1}, \ldots, x_{21}\right\}$, and all basic 


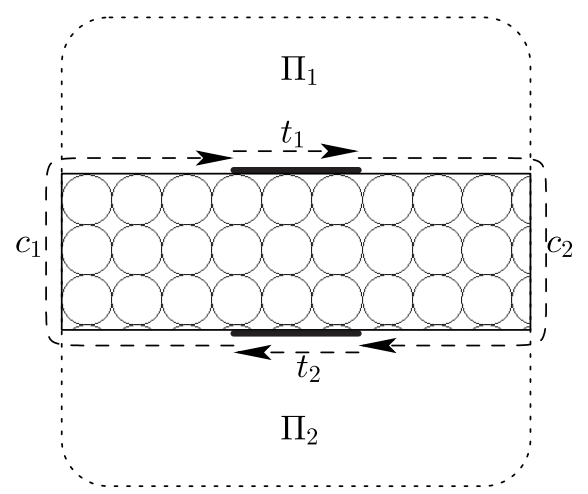

Figure 11. The subdiagram $\Delta^{\prime}$, Lemma 5.14 .

letters of $\ell\left(c_{2}\right)$ are in $\left\{x_{n-20}, \ldots, x_{n}\right\}$. Let $h_{1}=\ell\left(c_{1}^{-1}\right)$ and $h_{2}=\ell\left(c_{2}\right)$. Then $\left[h_{1}\right]=\left[w_{j}\right]\left[h_{2}\right]\left[w_{j}\right]^{-1}$. Let $F$ be the subgroup of $G$ generated by $\left\{a_{1}, \ldots, a_{21}\right\} \cup$ $\left\{a_{n-20}, \ldots, a_{n}\right\}$. By Property 1 , the group $F$ is free of rank 42 (since $42 \leq n-21$ ), and elements of $F$ are conjugate in $G$ if and only if they are conjugate in $F$. The elements $\left[h_{1}\right]$ and $\left[h_{2}\right]$ are in $F$. Moreover, $\left[h_{1}\right]$ belongs to the subgroup generated by $\left\{a_{1}, \ldots, a_{21}\right\}$, and $\left[h_{2}\right]$ belongs to the subgroup generated by $\left\{a_{n-20}, \ldots, a_{n}\right\}$. Since $\left[h_{1}\right]$ and $\left[h_{2}\right]$ are conjugate in $G$, they are conjugate in $F$ and therefore $\left[h_{1}\right]=\left[h_{2}\right]=1$. Hence, $\ell\left(c_{1} t_{1} c_{2} t_{2}\right)$ is freely trivial. This gives a contradiction.

Lemma 5.15. Let $\Pi_{1}$ and $\Pi_{2}$ be distinct faces of $\Delta$, both incident to selected external edges of $\Delta$. Suppose the rank of $\Pi_{1}$ is greater than the rank of every other face of $\Delta$. Let $s_{1}$ and $s_{2}$ be the maximal selected subpaths of $\partial \Pi_{1}$ and $\partial \Pi_{2}$, respectively. Suppose the label of one of the paths $s_{1}$ or $s_{2}$ is regular, and the label of the other is counter-regular. Let $s_{1}^{\prime}$ and $s_{2}^{\prime}$ be the maximal selected external oriented arcs of $\Delta$ that are subpaths of $s_{1}$ and $s_{2}$, respectively. Suppose there are at least 2 distinct basic letters in the label of each of the paths $s_{1}^{\prime}$ and $s_{2}^{\prime}$. Let $e_{11}$ and $e_{1 n}$ be respectively the initial and the terminal subpaths of $s_{1}$ of length $\left|s_{1}\right| / n$. Then at least one of the paths $e_{11}$ or $e_{1 n}$ has the property that every edge that lies on it is internal in $\Delta$ and does not lie on $s_{2}$.

Proof. Observe that it suffices to prove that at least one of the paths $e_{1 n}^{-1}$ or $e_{11}^{-1}$ has the property that every edge that lies on it is internal in the mirror copy of $\Delta$ and does not lie on $s_{2}^{-1}$.

Assume that $\ell\left(s_{1}\right)$ is counter-regular (if it is not, pass from $\Delta, b_{1}, b_{2}, l_{1}, l_{2}, s_{1}$, $s_{2}, s_{1}^{\prime}, s_{2}^{\prime}, e_{11}, e_{1 n}$ to the mirror copy of $\Delta, b_{2}^{-1}, b_{1}^{-1}, l_{1}^{-1}, l_{2}^{-1}, s_{1}^{-1}, s_{2}^{-1}, s_{1}^{\prime-1}, s_{2}^{\prime-1}$, $e_{1 n}^{-1}, e_{11}^{-1}$, respectively). Then $s_{1}^{\prime}$ is a subpath of $b_{1}^{-1}$, and $s_{2}^{\prime}$ is a subpath of $b_{2}^{-1}$.

Let $s_{1-}^{\prime}$ and $s_{1+}^{\prime}$ be the paths such that $s_{1}=s_{1-}^{\prime} s_{1}^{\prime} s_{1+}^{\prime}$. Let $t_{1}$ and $t_{2}$ be the paths such that $\left\langle s_{1} t_{1}\right\rangle=\partial \Pi_{1}$ and $\left\langle s_{2} t_{2}\right\rangle=\partial \Pi_{2}$. Let $q_{1}$ be the path such that $\left\langle s_{1}^{-1} q_{1}\right\rangle=\partial \Delta$. Let $\Delta^{\prime}$ be the disc subdiagram of $\Delta$ obtained by removing the face $\Pi_{1}$ and all edges that lie on $s_{1}^{\prime}$ together with all intermediate vertices of $s_{1}^{\prime}$. The contour of $\Delta^{\prime}$ is $\left\langle s_{1+}^{\prime} t_{1} s_{1-}^{\prime} q_{1}\right\rangle$. Let $e_{21}$ and $e_{2 n}$ be respectively the initial and terminal subpaths of $s_{2}$ of length $\left|s_{2}\right| / n$. 
Denote the ranks of $\Pi_{1}$ and $\Pi_{2}$ by $j_{1}$ and $j_{2}$, respectively. Then

$$
\begin{array}{cl}
\ell\left(s_{1}\right)=x_{n}^{-m_{j_{1}}} x_{n-1}^{-m_{j_{1}}} \cdots x_{1}^{-m_{j_{1}}}, & \ell\left(t_{1}\right)=w_{j_{1}}, \\
\ell\left(s_{2}\right)=x_{1}^{m_{j_{2}}} x_{2}^{m_{j_{2}}} \cdots x_{n}^{m_{j_{2}}}, & \ell\left(t_{2}\right)=w_{j_{2}}^{-1}, \\
\ell\left(e_{11}\right)=x_{n}^{-m_{j_{1}}}, \quad \ell\left(e_{1 n}\right)=x_{1}^{-m_{j_{1}}}, \quad \ell\left(e_{21}\right)=x_{1}^{m_{j_{2}}}, & \ell\left(e_{2 n}\right)=x_{n}^{m_{j_{2}}} .
\end{array}
$$

At most one of the two group letters $x_{1}^{ \pm 1}$ and at most one of $x_{n}^{ \pm 1}$ can occur as labels of oriented edges of $\partial \Delta$ (the labels of the representatives of $\partial \Delta$ are cyclically reduced). Therefore, at least one of the paths $e_{1 n}$ or $e_{21}$ and at least one of the paths $e_{11}$ or $e_{2 n}$ do not have external oriented edges in $\Delta$. By Lemma 5.11, all oriented edges of $e_{11}$ or all oriented edges of $e_{1 n}$ are internal in $\Delta$.

Suppose the conclusion of the lemma does not hold. Then some oriented edge of $e_{11}$ is either external in $\Delta$ or inverse to an oriented edge of $e_{2 n}$, and some oriented edge of $e_{1 n}$ is either external in $\Delta$ or inverse to an oriented edge of $e_{21}$. Therefore, as follows from Lemma 5.11, at least one of the following 3 cases takes place:

- some oriented edge of $e_{11}$ is inverse to an oriented edge of $e_{2 n}$, and some oriented edge of $e_{1 n}$ is inverse to an oriented edge of $e_{21}$, or

○ some oriented edge of $e_{11}$ is external in $\Delta$, and some oriented edge of $e_{1 n}$ is inverse to an oriented edge of $e_{21}$, or

- some oriented edge of $e_{11}$ is inverse to an oriented edge of $e_{2 n}$, and some oriented edge of $e_{1 n}$ is external in $\Delta$.

In every case the path $s_{2}$ has a common oriented edge with $s_{1-}^{\prime-1}$ or $s_{1+}^{\prime-1}$. Let $\tilde{s}_{2}$ be the maximal common nontrivial subpath of $s_{2}$ and $\left(s_{1-}^{\prime} q_{1} s_{1+}^{\prime}\right)^{-1}$ (see Lemma 5.10). Now, consider each of the 3 cases separately (see Figure 12).

(Notice that Case 3 is "symmetric" with Case 2.)

Case 1: some oriented edge of $e_{11}$ is inverse to an oriented edge of $e_{2 n}$, and some oriented edge of $e_{1 n}$ is inverse to an oriented edge of $e_{21}$. Then some nontrivial initial subpath of $\tilde{s}_{2}^{-1}$ is a terminal subpath of $s_{1-}^{\prime}$, and some nontrivial terminal subpath of $\tilde{s}_{2}^{-1}$ is an initial subpath of $s_{1+}^{\prime}$ (because $\tilde{s}_{2}^{-1}$ is a subpath of $s_{1-}^{\prime} q_{1} s_{1+}^{\prime}$ ). Let $v_{1}$ and $v_{2}$ be the paths such that $v_{1} q_{1} v_{2}=\tilde{s}_{2}^{-1}$. Then $v_{1}$ is a nontrivial terminal subpath of $s_{1-}^{\prime}$, and $v_{2}$ is a nontrivial initial subpath of $s_{1+}^{\prime}$. The label of $s_{1-}^{\prime} q_{1} s_{1+}^{\prime}$ is counter-regular since the labels of $s_{1-}^{\prime}, v_{1} q_{1} v_{2}$, and $s_{1+}^{\prime}$ are such. As it is assumed, $\operatorname{rank}(\Pi)<j_{1}$ for every face $\Pi$ of $\Delta^{\prime}$. Recall that $\left\langle s_{1+}^{\prime} t_{1} s_{1-}^{\prime} q_{1}\right\rangle=\partial \Delta^{\prime}$. Hence, the group word $w_{j_{1}}=\ell\left(t_{1}\right)$ equals the regular word $\ell\left(s_{1-}^{\prime} q_{1} s_{1+}^{\prime}\right)^{-1}$ modulo the relations $r=1, r \in \mathcal{R}_{j_{1}-1}$. This contradicts the choice of $w_{j_{1}}$.

Case 2: some oriented edge of $e_{11}$ is external in $\Delta$, and some oriented edge of $e_{1 n}$ is inverse to an oriented edge of $e_{21}$. Then $s_{1-}^{\prime}$ is a proper initial subpath of $e_{11}$. Let $v_{1}$ be the initial subpath of $s_{1}^{\prime}$ such that $s_{1-}^{\prime} v_{1}=e_{11}$. Then $v_{1}^{-1}$ is a subpath of $l_{2}$ since $\ell\left(v_{1}^{-1}\right)$ is a nontrivial power of $x_{n}$. Some nontrivial terminal subpath of $\tilde{s}_{2}^{-1}$ is an initial subpath of $s_{1+}^{\prime}$. Therefore, $s_{2}^{\prime-1}$ is a terminal subpath of $q_{1}$, and $v_{1}^{-1} q_{1}$ is a subpath of $b_{2}$ (because $s_{2}^{\prime-1}$ is a common subpath of $q_{1}$ and $b_{2}$ ). Let $v_{2}$ be the path such that $s_{2}^{\prime-1} v_{2}$ is a terminal subpath of $\tilde{s}_{2}^{-1}$. Then $v_{2}$ is a nontrivial initial subpath of $s_{1+}^{\prime}$. The label of $q_{1} s_{1+}^{\prime}$ is counter-regular since the labels of $q_{1}, s_{2}^{\prime-1} v_{2}$, and $s_{1+}^{\prime}$ are such. The reduced form of $\ell\left(s_{1-}^{\prime} q_{1} s_{1+}^{\prime}\right)$ is counter-regular since $\ell\left(s_{1-}^{\prime}\right)$ is a power of $x_{n}$, and $\ell\left(q_{1} s_{1+}^{\prime}\right)$ is counter-regular. As it is assumed, $\operatorname{rank}(\Pi)<j_{1}$ for every face $\Pi$ of $\Delta^{\prime}$. Hence, the group word $w_{j_{1}}=\ell\left(t_{1}\right)$ equals a regular word 


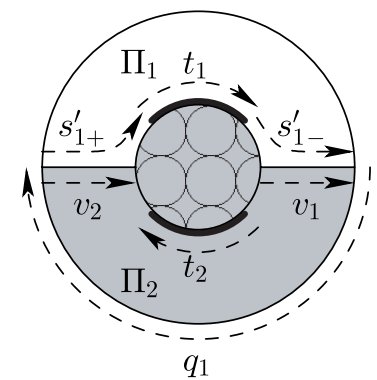

\section{Case 1}

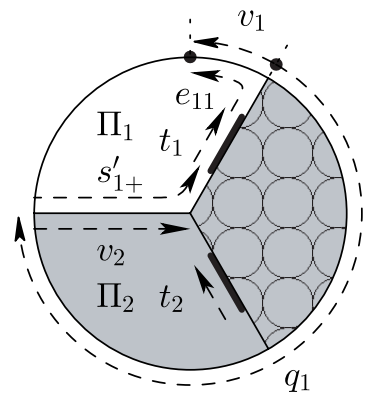

Case 2

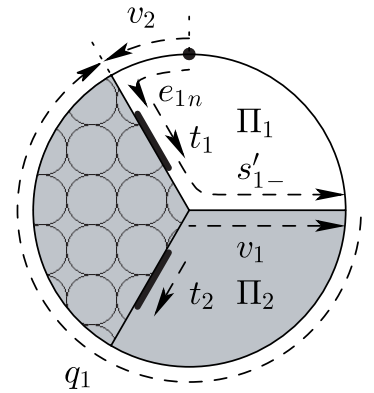

Case 3

Figure 12. Cases 1-3, Lemma 5.15.

(the reduced form of $\ell\left(s_{1-}^{\prime} q_{1} s_{1+}^{\prime}\right)^{-1}$ ) modulo the relations $r=1, r \in \mathcal{R}_{j_{1}-1}$. This contradicts the choice of $w_{j_{1}}$.

Case 3: some oriented edge of $e_{11}$ is inverse to an oriented edge of $e_{2 n}$, and some oriented edge of $e_{1 n}$ is external in $\Delta$. Contradiction is obtained as in Case 2.

No other case is possible. Thus, at least one of the paths $e_{11}$ or $e_{1 n}$ has no common oriented edges with $s_{2}^{-1}$ and no external oriented edges.

Lemma 5.16. Let $\Pi_{1}, \Pi_{2}, s_{1}, s_{1}^{\prime}, s_{1-}^{\prime}, s_{1+}^{\prime}, s_{2}, s_{2}^{\prime}, s_{2-}^{\prime}, s_{2+}^{\prime}, z_{1}, z_{2}$ be such as in Lemma 5.12. Let $c_{1}=s_{2-}^{\prime} z_{1} s_{1+}^{\prime}$ and $c_{2}=s_{1-}^{\prime} z_{2} s_{2+}^{\prime}$. Then $\Delta$ has a simple disc S-subdiagram $\Phi$ such that $\Pi_{1}$ and $\Pi_{2}$ are not in $\Phi$, and the total number of selected external edges of $\Phi$ that lie on $c_{1}$ or $c_{2}$ is greater than

$$
\frac{n-21}{n} \sum_{\Pi \in \Phi(2)}|\partial \Pi| \text {. }
$$

Proof. Observe that to prove this lemma, it suffices to prove the analogous lemma for the mirror copy of $\Delta$.

By Lemma 5.14, $\operatorname{rank}\left(\Pi_{1}\right) \neq \operatorname{rank}\left(\Pi_{2}\right)$. If $\operatorname{rank}\left(\Pi_{1}\right)<\operatorname{rank}\left(\Pi_{2}\right)$, pass from $\Delta$, $\Pi_{1}, \Pi_{2}, s_{1}, s_{1-}^{\prime}, s_{1}^{\prime}, s_{1+}^{\prime}, s_{2}, s_{2-}^{\prime}, s_{2}^{\prime}, s_{2+}^{\prime}, z_{1}, z_{2}, c_{1}, c_{2}$ to the mirror copy of $\Delta$, $\Pi_{2}, \Pi_{1}, s_{2}^{-1}, s_{2+}^{\prime-1}, s_{2}^{\prime-1}, s_{2-}^{\prime-1}, s_{1}^{-1}, s_{1+}^{\prime-1}, s_{1}^{\prime-1}, s_{1-}^{-1}, z_{1}^{-1}, z_{2}^{-1}, c_{1}^{-1}, c_{2}^{-1}$, respectively. Hence, assume that $j_{1}=\operatorname{rank}\left(\Pi_{1}\right)>\operatorname{rank}\left(\Pi_{2}\right)=j_{2}$.

Let $t_{1}$ and $t_{2}$ be the paths such that $\left\langle s_{1} t_{1}\right\rangle=\partial \Pi_{1}$ and $\left\langle s_{2} t_{2}\right\rangle=\partial \Pi_{2}$. Let $\Delta^{\prime}$ be the disc S-subdiagram of $\Delta$ obtained by removing the faces $\Pi_{1}$ and $\Pi_{2}$ and all edges that lie on $s_{1}^{\prime}$ or $s_{2}^{\prime}$ together with all intermediate vertices of $s_{1}^{\prime}$ and $s_{2}^{\prime}$. The contour of $\Delta^{\prime}$ is $\left\langle c_{1} t_{1} c_{2} t_{2}\right\rangle$. By Lemma 5.13, the diagram $\Delta^{\prime}$ is nondegenerate. 
Consider 2 cases:

Case 1: there is a face $\Pi$ in $\Delta^{\prime}$ such that $\operatorname{rank}(\Pi) \geq \operatorname{rank}\left(\Pi_{1}\right)$. Then let $\Phi$ be the maximal simple disc S-subdiagram of $\Delta^{\prime}$ that contains such a face $\Pi$. Let $\Sigma$ be the sum of the degrees of all the faces of $\Phi$. Then $\Sigma \geq|\partial \Pi| \geq\left|\partial \Pi_{1}\right|>\left|\partial \Pi_{2}\right|$. By Lemma 5.3, the number of selected external edges of $\Phi$ is at least $(1-2 \mu) \Sigma$. Therefore, the number of selected external edges of $\Phi$ that do not lie on $t_{1}$ nor on $t_{2}$ is at least

$$
(1-2 \mu) \Sigma-\lambda_{1}\left|\partial \Pi_{1}\right|-\lambda_{1}\left|\partial \Pi_{2}\right|>\left(1-2 \mu-2 \lambda_{1}\right) \Sigma>\frac{n-21}{n} \Sigma .
$$

Case 2: the rank of every face of $\Delta^{\prime}$ is less than the rank of $\Pi_{1}$. Let $e_{11}$ and $e_{1 n}$ be respectively the initial and the terminal subpaths of $s_{1}$ of length $m_{j_{1}}=\left|s_{1}\right| / n$. By Lemma 5.15, at least one of the paths $e_{11}$ or $e_{1 n}$ has the property that every edge that lies on it either is incident to a face of $\Delta^{\prime}$ or lies on $t_{1}$ or $t_{2}$. Let $e$ be one of the paths $e_{11}$ or $e_{1 n}$ with this property.

Since

$$
\left|t_{1}\right|+\left|t_{2}\right|=\left|w_{j_{1}}\right|+\left|w_{j_{2}}\right|<\frac{1}{2} m_{j_{1}}+\frac{1}{2} m_{j_{1}}=m_{j_{1}}=|e|
$$

(see (5.5)), the ratio of the number of edges that lie on $t_{1}$ or $t_{2}$ and are incident with faces of $\Delta^{\prime}$ (consequently, do not lie on $e$ ) to the number of edges that lie on $e$ and are incident with faces of $\Delta^{\prime}$ (equivalently, lie neither on $t_{1}$ nor on $t_{2}$ ) is at most

$$
\frac{\left|t_{1}\right|+\left|t_{2}\right|}{|e|}=\frac{\left|w_{j_{1}}\right|+\left|w_{j_{2}}\right|}{m_{j_{1}}} \leq \frac{2 n \lambda_{1}}{1-\lambda_{1}}
$$

(see (5.4)). Let $\Phi$ be a maximal simple disc S-subdiagram of $\Delta^{\prime}$ such that the ratio of the number of its (external) edges that lie on $t_{1}$ or $t_{2}$ to the number of its (external) edges that lie on $e$ is at most

$$
\frac{2 n \lambda_{1}}{1-\lambda_{1}}
$$

Let $\Sigma$ be the sum of the degrees of all the faces of $\Phi$. Since all the edges that lie on $e$ are labelled with a same basic letter, the number of edges of $\Phi$ that lie on $e$ is less than

$$
\left(\lambda_{1}+\frac{1-\lambda_{1}}{n}\right) \Sigma
$$

By Lemma 5.3, the number of selected external edges of $\Phi$ is at least $(1-2 \mu) \Sigma$. Therefore, the number of selected external edges of $\Phi$ that neither lie on $t_{1}$ nor on $t_{2}$ is greater than

$$
\left(1-2 \mu-\frac{2 n \lambda_{1}}{1-\lambda_{1}}\left(\lambda_{1}+\frac{1-\lambda_{1}}{n}\right)\right) \Sigma=\left(1-2 \mu-2 \lambda_{1}-\frac{2 n \lambda_{1}^{2}}{1-\lambda_{1}}\right) \Sigma \geq \frac{n-21}{n} \Sigma
$$

$($ see $(5.2))$.

Every external edge of $\Phi$ that neither lies on $t_{1}$ nor on $t_{2}$ lies on $c_{1}$ or $c_{2}$. Thus, the desired inequality holds in the both cases.

Proof of Property 2. The existence is obvious from the choice of relators.

Suppose that presentation of some element of $G$ in the form $a_{1}^{k_{1}} \cdots a_{n}^{k_{n}}$ is not unique. Then there exist two distinct regular group words over $\mathfrak{A}$ whose values in $G$ coincide. Consider such a pair of group words with the minimal sum of their lengths. Consider a minimal deduction diagram for the equality of these group words over $\langle\mathfrak{A} \| \mathcal{R}\rangle$. More precisely, let $\Delta$ be a special disc S-diagram over $\langle\mathfrak{A} \| \mathcal{R}\rangle$ 
whose contour has a representative of the form $p_{1} p_{2}$ such that $\ell\left(p_{1}\right)$ and $\ell\left(p_{2}^{-1}\right)$ are distinct regular group words, and $\ell\left(p_{1} p_{2}\right)$ is cyclically reduced; moreover, let $\Delta$ be such an S-diagram with the minimal possible number of faces.

Choose faces $\Pi_{1}$ and $\Pi_{2}$ and paths $s_{1}, s_{1}^{\prime}, s_{1-}^{\prime}, s_{1+}^{\prime}, s_{2}, s_{2}^{\prime}, s_{2-}^{\prime}, s_{2+}^{\prime}, z_{1}, z_{2}$ as in Lemma 5.12. Denote the paths $s_{2-}^{\prime} z_{1} s_{1+}^{\prime}$ and $s_{1-}^{\prime} z_{2} s_{2+}^{\prime}$ by $c_{1}$ and $c_{2}$, respectively (as in the hypotheses of Lemma 5.16). Apply Lemma 5.16: let $\Phi$ be a simple disc S-subdiagram of $\Delta$ such that the number of selected external edges of $\Phi$ that lie on $c_{1}$ or $c_{2}$ is greater than

$$
\frac{n-21}{n} \sum_{\Pi \in \Phi(2)}|\partial \Pi| .
$$

By the choice of $\Pi_{1}$ and $\Pi_{2}$, all basic letters of $\ell\left(c_{1}\right)$ are in $\left\{x_{1}, \ldots, x_{21}\right\}$, and all basic letters of $\ell\left(c_{2}\right)$ are in $\left\{x_{n-20}, \ldots, x_{n}\right\}$. Therefore, by Proposition 5.2, the number of selected external edges of $\Phi$ that lie on $c_{1}$ or $c_{2}$ is less than

$$
\frac{42}{n} \sum_{\Pi \in \Phi(2)}|\partial \Pi| .
$$

Since $n-21 \geq 42$, one has a contradiction. The uniqueness is proved.

Remark 5.1. The "symmetry" mentioned in the proofs of Lemmas 5.8, 5.10, 5.13, and 5.15 is the symmetry between the S-diagram $\Delta$ and the S-diagram obtained from the mirror copy of $\Delta$ by re-labelling according to the following rule: if the label of an oriented edge edge $e$ is $x_{i}^{\sigma}$, then re-label $e$ with $x_{n+1-i}^{-\sigma}$ (this gives a diagram not over the same presentation).

The proof of the next property uses a homological argument.

Property 3. The group $G$ is torsion-free.

Proof. The group presentation $\langle\mathfrak{A} \| \mathcal{R}\rangle$ is strongly aspherical by Corollary 2 of Proposition 5.1; therefore, it is aspherical in the sense of O1. Since no element of $\mathcal{R}$ represents a proper power in the free group on $\mathfrak{A}$, the relation module $M$ of $\langle\mathfrak{A} \| \mathcal{R}\rangle$ is a free $G$-module by Corollary 32.1 in [01]. Therefore, there exists a finite-length free resolution of $\mathbb{Z}$ over $\mathbb{Z} G$ :

$$
0 \rightarrow M \rightarrow \bigoplus_{x \in \mathfrak{A}} \mathbb{Z} G \rightarrow \mathbb{Z} G \rightarrow \mathbb{Z} \rightarrow 0
$$

where $\mathbb{Z} G$ and $\bigoplus_{x \in \mathfrak{A}} \mathbb{Z} G$ are identified with the $G$-modules of, respectively, 0- and 1-dimensional cellular chains of the Cayley complex of $\langle\mathfrak{A} \| \mathcal{R}\rangle$ (see $[\mathrm{Br}]$ ).

Now suppose that $G$ has torsion. Let $H$ be a nontrivial finite cyclic subgroup of $G$. Every free $G$-module may be naturally regarded as a free $H$-module. Hence, the resolution (5.6) may be viewed as a free resolution of $\mathbb{Z}$ over $\mathbb{Z} H$. This contradicts the fact that all odd-dimensional homology groups of $H$ are nontrivial.

For more results on group presentations with various forms of asphericity, and torsion in such groups, see [ChCoH], [H1, [H2, and Theorems 32.1, 32.2 in [01.

Properties 1, 2, and 3 of $G$ demonstrate that the answer to Bludov's question is negative.

Remark 5.2. If $n$ was chosen to be less than 63 but greater than $26(26<n<63)$, then Properties 1 and 3 would still hold, but the proof of Property 2 would not work. 
For every $i \in \mathbb{N} \cup\{0\}$, let $G_{i}$ be the group defined by the presentation $\left\langle\mathfrak{A} \| \mathcal{R}_{i}\right\rangle$ (see Section 2). Also for every $i \in \mathbb{N} \cup\{0\}$, let $\phi_{i}$ be the natural epimorphism $G_{i} \rightarrow G_{i+1}$.

Property 4. All groups $G_{0}, G_{1}, G_{2}, \ldots$ are hyperbolic, and $G$ is isomorphic to the direct limit of $G_{0} \stackrel{\phi_{0}}{\longrightarrow} G_{1} \stackrel{\phi_{1}}{\longrightarrow} G_{2} \stackrel{\phi_{2}}{\longrightarrow} \cdots$.

Proof. Obviously, $G$ is isomorphic to the direct limit of $G_{0} \stackrel{\phi_{0}}{\longrightarrow} G_{1} \stackrel{\phi_{1}}{\longrightarrow} G_{2} \stackrel{\phi_{2}}{\longrightarrow} \cdots$. The groups $G_{0}, G_{1}, G_{2}, \ldots$ are hyperbolic by the Corollary of Proposition 5.3.

It is easy to see that every recursively presented group with a finite regular file basis has solvable word problem. (Note that if a finitely generated group has a recursive presentation with a countably infinite alphabet, then it also has a recursive presentation with a finite alphabet.) In particular, if the group $G$ is recursively presented, then it has solvable word problem. Generally, a recursively presented finitely generated group $H$ has solvable word problem if an only if there exists a recursive set of group words $X$ in a finite alphabet $\mathfrak{B}$ such that relative to some mapping of $\mathfrak{B}$ to $H$, every element of $H$ has a unique representative in $X$. In the case of $G$, the set of all regular group words over $\mathfrak{A}$ may be taken as such a recursive set of representatives.

Property 5 (conditional). If $G$ is recursively presented, then it has solvable conjugacy problem.

Proof. Assume $G$ is recursively presented. Then, as noted above, $G$ has solvable word problem.

Let $q$ be an integer such that $q \geq 1 /(1-2 \mu)$.

Here is a description of an algorithm that solves the conjugacy problem in $G$ :

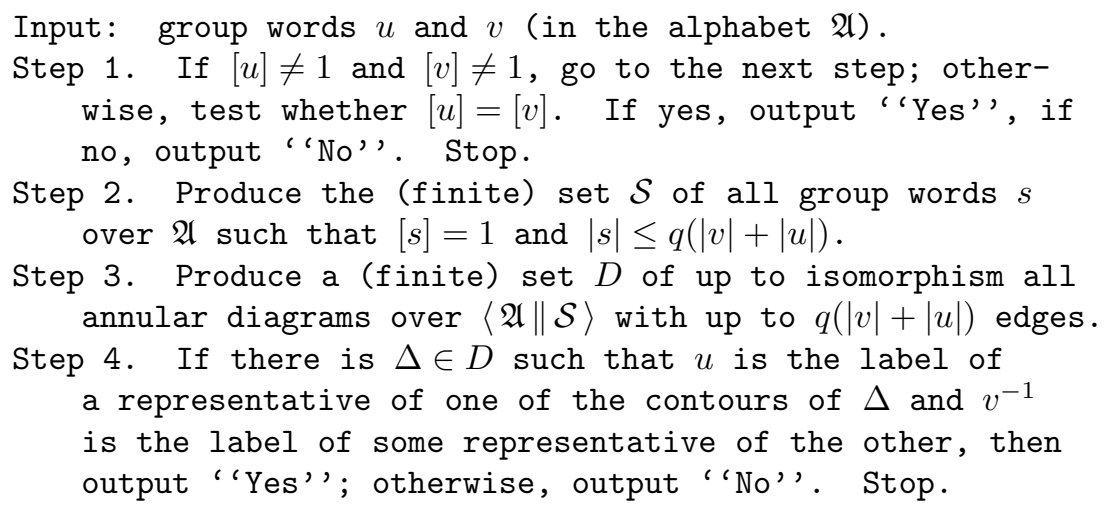

On an input $(u, v)$, this algorithm gives "Yes" as the output if and only if $[u]$ and $[v]$ are conjugate in $G$; otherwise, it gives "No." Below is an outline of a proof.

It is clear that the algorithm gives "Yes" only if $[u]$ and $[v]$ are conjugate in $G$. It is also clear that on every input it gives either "Yes" or "No," and terminates.

Now, let $u$ and $v$ be arbitrary group words representing conjugate elements of $G$, and consider $(u, v)$ as an input to the algorithm.

Case 1: $[u]=1$ or $[v]=1$. Then $[u]=1=[v]$, and therefore the algorithm gives "Yes" on Step 1.

Case 2: $[u] \neq 1$ and $[v] \neq 1$. Let $\Delta$ be a weakly reduced annular disc diagram over $\langle\mathfrak{A} \| \mathcal{R}\rangle$ such that $u$ and $v^{-1}$ are the labels of some representatives of its two 
contours (such $\Delta$ exists by Lemma V.5.2 in LyS). Let $\mathcal{S}$ be the set of group words produced by the algorithm on Step 2. Every element of $\mathcal{R}$ of length at most $q(|v|+|u|)$ is in $\mathcal{S}$. Let $D$ be the set of annular diagrams produced by the algorithm on Step 3. By Proposition 5.1 and the Main Lemma,

$$
|u|+|v| \geq(1-2 \mu) \sum_{\Pi \in \Delta(2)}|\partial \Pi| \geq \frac{1}{q} \sum_{\Pi \in \Delta(2)}|\partial \Pi|
$$

(recall that a special selection exists on every diagram over $\langle\mathfrak{A} \| \mathcal{R}\rangle$ ). Therefore, the length of the contour of every face of $\Delta$ is at most $q(|u|+|v|)$, and the total number of edges of $\Delta$ is

$$
\begin{aligned}
\|\Delta(1)\|=\frac{1}{2}\left(\sum_{\Pi \in \Delta(2)}|\partial \Pi|+|u|+|v|\right) \leq \frac{1}{2}(q(|u| & +|v|)+|u|+|v|) \\
& =\frac{q+1}{2}(|u|+|v|) \leq q(|u|+|v|) .
\end{aligned}
$$

Hence, $\Delta$ is isomorphic to some element of $D$, and the algorithm returns "Yes" on Step 4 .

Proof of the theorem (see the Introduction). Let $N$ be an integer such that $\lambda_{1} n N \geq 1$. Let the construction in Section 2 be carried out under the following two additional conditions:

(1) the order imposed on the set of all reduced group words is deg-lex;

(2) for every $i$ for which $r_{i}$ is defined, $m_{i}=N\left|w_{i}\right|+i$.

Evidently, these conditions are consistent with the rest.

By Proposition 5.4, the group $G$ is recursively presented. Properties 1-5 show that up to isomorphism $G$ is a desired group. ( $G$ is isomorphic to a direct limit of a sequence of hyperbolic groups with respect to a family of surjective homomorphisms, but a desired group must be such a limit.)

\section{Comments}

The group $G$ and its presentation $\langle\mathfrak{A} \| \mathcal{R}\rangle$ mentioned in this section are defined in Section 2.

Proposition 6.1. No hyperbolic group can be used as an example to demonstrate the negative answer to Bludov's question.

Proof. Suppose a hyperbolic group $H$ is an example demonstrating the negative answer. Then $H$ is boundedly generated (is the product of a finite sequence of its cyclic subgroups) and not virtually polycyclic. In particular, $H$ is non-elementary. Corollary 4.3 in [Mi] states that every boundedly generated hyperbolic group is elementary. (This fact also follows from Corollary 2 in $\mathrm{O} 2$.) This gives a contradiction.

Corollary. The group $G$ is not finitely presented.

Proof. Suppose $G$ is finitely presented. Then the presentation $\langle\mathfrak{A} \| \mathcal{R}\rangle$ is finite. By Corollary of Proposition 5.3, $G$ is hyperbolic. This contradicts Proposition 6.1.

Thus, the set $\mathcal{R}$ and the sequences $\left\{w_{i}\right\}_{i=1, \ldots},\left\{m_{i}\right\}_{i=1, \ldots},\left\{r_{i}\right\}_{i=1, \ldots}$ constructed in Section 2 are infinite. 


\section{ACKNOWLEDGEMENTS}

The author thanks Alexander Ol'shanskiy for suggesting the problem and engaging in fruitful discussions during the course of this work.

\section{REFERENCES}

[ABC+] Alonso, Juan M.; Brady, Thomas; Cooper, Daryl; Ferlini, Vincent; Lustig, Martin; Mihalik, Michael L.; Shapiro, Michael; Short, Hamish B., Notes on word hyperbolic groups, Group theory from a geometrical viewpoint, (proceedings of the workshop held in Trieste, March 26 - April 6, 1990, World Sci. Publ., 1991, pp. 3-63. MR.1170363 (93g:57001)

[Bl] Bludov, Vasiliy Vasilievich, File bases in groups, Algebra and Logic 34 (3) (1995), 131139 transl. from Russian. MR:1364464 (97b:20043)

[Br] Brown, Kenneth S., Cohomology of groups, corrected reprint of the 1982 original, Springer-Verlag, 1994. MR1324339(96a:20072)

[ChCoH] Chiswell, Ian M.; Collins, Donald J.; Huebschmann, Johannes, Aspherical group presentations, Math Z. 178 (1) (1981), 1-36. MR0627092 (83a:20046)

[H1] Huebschmann, Johannes, Cohomology theory of aspherical groups and of small cancellation groups, J. Pure Appl. Algebra 14 (2) (1979), 137-143. MR0524183 (80e:20064)

[H2] - The homotopy type of a combinatorially aspherical presentation, Math Z 173 (2) (1980), 163-169. MR0583383 (81m:57003)

[I] Itô, Noboru, Über das Produkt von zwei abelschen Gruppen, Math. Z. 62 (1955), 400-401 (German). MR0071426 (17:125b)

[kn] The Kourovka Notebook, unsolved problems in group theory, 13th augmented edition, Russian Acad. of Sci. Siber. Div., Inst. Math., Novosibirsk, 1995 transl. from Russian. MR1392713 (97d:20001)

[LeR] Lennox, John C.; Roseblade, James E., Soluble products of polycyclic groups, Math. Z. 170 (2) (1980), 153-154. MR0562584 (81a:20047)

[LyS] Lyndon, Roger C.; Schupp, Paul E., Combinatorial group theory, reprint of the 1977 edition, Springer-Verlag, Berlin, 2001. MR.1812024 (2001i:20064)

[Mi] Minasyan, Ashot, On products of quasiconvex subgroups in hyperbolic groups, Internat. J. Algebra Comput. 14 (2) (2004), 173-195. MR2058319(2005a:20063)

$[\mathrm{Mu}]$ Muranov, Alexey Yu., Diagrams with selection and method for constructing boundedly generated and boundedly simple groups, Comm. Algebra 33 (4) (2005), 1217-1258. MR2136699 (2006b:20044)

[O1] Ol'shanskii, Alexander Yu., Geometry of defining relations in groups, Kluwer Academic Publishers, Dordrecht, Boston, 1991 transl. from Russian. MR.1191619 (93g:20071)

[O2] On residualing homomorphisms and G-subgroups of hyperbolic groups, Internat. J. Algebra Comput. 3 (4) (1993), 365-409. MR1250244 (94i:20069)

Department of Mathematics, Vanderbilt University, 1326 Stevenson Center, NashVILle, Tennessee 37240-0001

Current address: Institut Camille Jordan, Université Lyon 1, 43 blvd du 11 novembre 1918, 69622 Villeurbanne cedex, France

E-mail address: muranov@math.univ-lyon1.fr 